LBNL-45871

\title{
Semianalytical Solutions of RADIOACTIVE OR REACTIVE TRANSPORT IN VARIABLY-FRACTURED LAYERED MEdia: 1. Solutes
}

\section{George J. Moridis}

\section{Earth Sciences Division}

Lawrence Berkeley National Laboratory

Berkeley, CA 94720

\section{October 2001}

This work was supported by the Director, Office of Civilian Radioactive Waste Management, U.S. Department of Energy, through Memorandum Purchase Order EA9013MC5X between Bechtel SAIC Company. LLC and the Ernest Orlando Lawrence Berkeley National Laboratory (Berkeley Lab). The support is provided to Berkeley Lab through the U.S. Department of Energy Contract No. DE-AC03-76SF00098. 


\title{
Semianalytical Solutions of Radioactive or Reactive Transport in Variably-Fractured Layered Media: 1. Solutes
}

\begin{abstract}
In this paper, semianalytical solutions are developed for the problem of transport of radioactive or reactive solute tracers through a layered system of heterogeneous fractured media with misaligned fractures. The tracer transport equations in the nonflowing matrix account for (a) diffusion, (b) surface diffusion, (c) mass transfer between the mobile and immobile water fractions, (d) linear kinetic or equilibrium physical, chemical, or combined solute sorption or colloid filtration, and (e) radioactive decay or first-order chemical reactions. The tracer-transport equations in the fractures account for the same processes, inadditiontoadvectionandhydrodynamicdispersion. Anynumberofradioactive decay daughter products (or products of a linear, first-order reaction chain) can be tracked. The solutions, which are analytical in the Laplace space, are numerically inverted to provide the solution in time and can accommodate any number of fractured and/or porous layers. The solutions are verified using analytical solutions for limiting cases of solute and colloid transport through fractured and porous media. The effect of important parameters on the transport of ${ }^{3} \mathrm{H},{ }^{237} \mathrm{~Np}$ and ${ }^{239} \mathrm{Pu}$ (and its daughters) is investigated in several test problems involving layered geological systems of varying complexity.
\end{abstract}

\section{Introduction}

The study of radioactive and/or reactive contaminant transport in complex fractured geologic systems has become increasingly important in recent years because of the need to predict the migration and fate of the contaminants. Currently, there are some very 
large contaminated sites (such as Hanford, Washington; Nevada Test Site (NTS), Nevada; Idaho National Engineering and Environmental Laboratory (INEEL), Idaho) where severe pollution by radioactive materials extends over large areas within the subsurface rocks.

At Yucca Mountain (YM), Nevada, the site of the potential repository for high-level nuclear waste, the transport of radioactive contaminants must be predicted for tens to hundreds of thousands of years. Performing reliable radionuclide transport calculations for this temporal and spatial scale is obviously very difficult, and furthermore it is impossible to verify the results. In addition, the complex geology of the site and the unsaturated nature of a significant portion of the flow path add to the difficulty in making such predictions.

The potential site is located in southern Nevada about $120 \mathrm{~km}$ northwest of Las Vegas, and is characterized by a thick unsaturated zone $(600-700 \mathrm{~m})$ and the presence of rocks onto which important radionuclides in the wastes tend to sorb strongly. The YM stratigraphy consists of layers of welded and nonwelded tuffs (with vastly different hydraulic, transport, and geochemical properties), with the former generally being extensively fractured and the latter behaving similarly to a porous medium [Montazer and Wilson, 1984; Liu et al., 1998; Bandurraga and Bodvarsson, 1999].

The varied geological and hydrological characteristics of the different tuff layers at Yucca Mountain make the modeling of flow and transport a challenging task. A single representation for all of the hydrogeologic units is inappropriate, and several different approaches and algorithms must be employed for obtaining reliable modeling results. Analytical and semianalytical models of transport that can account for the site heterogeneity are important because they allow the validation of complex multidimensional numerical models, are computationally efficient, and can provide bounding estimates of the possible solutions of the expected transport at the site.

Previous analyticalsolutions of solutetransport infractured mediainvolvedexclusively single semi-infinite domains (layers). Tang et al. [1981] developed a quasi two-dimensional solution for the transport of solutes in a single saturated fracture (i.e., with a semi-infinite 
matrix) that assumed a constant concentration boundary and accounted for (a) advection and dispersion in the fractures, (b) diffusion in the matrix, the fractures, and across their interface, (c) sorption onto the matrix and the fractures, and (d) radioactive decay. The analytical solution of Sudicky andFrind [1982] accounted forthe sameprocesses inasystem of parallel fractures (i.e., with a finite matrix block size). The solution of Robinson et al. [1998] is an extension of the Sudicky and Frind [1982] solution and accounts for the effect of fracture skin on transport in a system of parallel fractures. By neglecting hydrodynamic dispersion inthe fracturesand assuming aninstantaneous (Dirac-type) deposition of a parent radionuclide at the boundary, Sudicky and Frind [1984] obtained analytical solutions to the problem of transport of a two-member radioactive chain in a single fracture.

In this paper, semianalytical solutions are developed for the problem of transport of radioactive or reactive solute tracers (i.e., at concentrations that do not affect the fluid properties) through a layered system of heterogeneous fractured media with misaligned fractures (such as the unsaturated zone at YM). The solutions allow any number and combination of fractured and/or porous layers that can vary in hydraulic and transport properties, fracture frequency, water saturation, fracture flow, and fracture-matrix interaction. The tracer transport equations in the non-flowing matrix account for (a) molecular diffusion, (b) surface diffusion, (c) mass transfer between the mobile and immobile water fractions, (d) linear kinetic or equilibrium physical, chemical or combined solute sorption, and (e) radioactive decay or first-order chemical reactions. The solute transport equations in the fractures account for the same processes, in addition to advection and hydrodynamic dispersion. Any number of daughter products of radioactive decay (or of a linear, first-order reaction chain) can be tracked, and several boundary conditions can be accommodated.

\section{Solute Transport Equations}

\subsection{The PDE of Solute Transport}

The one-dimensional (1-D) Partial Differential Equation (PDE) of transport of a 
radioactiveorreactivesolutetracersthroughavariablysaturatedporous orfracturedmedium (PM or FM) is described by the equation

$$
\begin{aligned}
& D_{m} \frac{\partial^{2} C}{\partial x^{2}}+D_{i} \frac{\partial^{2} C_{i}}{\partial x^{2}}+D_{F} \frac{\partial^{2} F}{\partial x^{2}}-U \frac{\partial C}{\partial x} \\
& =\phi\left(S-S_{r}\right)\left(\frac{\partial C}{\partial t}+\delta_{r} \frac{\partial \mathcal{R}}{\partial t}\right)+\phi S_{r}\left(\frac{\partial C_{i}}{\partial t}+\delta_{r} \frac{\partial \mathcal{R}_{i}}{\partial t}\right)+(1-\phi) \rho \frac{\partial F}{\partial t} \\
& \quad+\lambda \delta_{\lambda}\left[\phi\left(S-S_{r}\right) C+\phi S_{r} C_{i}+(1-\phi) \rho F\right]
\end{aligned}
$$

where

$C$ dissolved species concentration in the mobile pore water $\left[M L^{-3}\right]$;

$D_{m} \quad$ intrinsic diffusion coefficient for the mobile pore water $\left[L^{2} T^{-1}\right]$;

$C_{i} \quad$ dissolved species concentration in the immobile pore water $\left[M L^{-3}\right]$;

$D_{i} \quad$ intrinsic diffusion coefficient in the immobile pore water $\left[L^{2} T^{-1}\right]$;

$F \quad=F_{p}+F_{c}$

$F_{p} \quad$ relative concentration of the physically adsorbed species $\left[\left(M L^{-3}\right) /\left(M L^{-3}\right)\right]$;

$F_{c} \quad$ relative concentration of the chemically sorbed species $\left[\left(M L^{-3}\right) /\left(M L^{-3}\right)\right]$;

$\mathcal{R}$ reacted species mass per unit volume in the mobile fraction $\left[M L^{-3}\right]$;

$\mathcal{R}_{i} \quad$ reacted species mass per unit volume in the immobile fraction $\left[M L^{-3}\right]$;

$D_{F} \quad$ apparent surface diffusion coefficient $\left[M L^{-1} T^{-1}\right]$;

$U=V \phi\left(S-S_{r}\right)$, Darcy velocity $\left[L T^{-1}\right]$

$V \quad$ pore flow velocity $\left[L T^{-1}\right]$;

$S \quad$ water saturation $\left[L^{3} / L^{3}\right]$;

$S_{r} \quad$ irreducible water saturation $\left[L^{3} / L^{3}\right]$;

$\rho \quad$ PM grain density $\left[M L^{-3}\right]$;

$\phi \quad$ total PM porosity $\left[L^{3} / L^{3}\right]$;

$\lambda \quad=\ln 2 / T_{1 / 2}$, radioactive decay constant $\left[T^{-1}\right] ;$

$T_{1 / 2}$ half-life of radioactive species $[T]$.

The parameters $\delta_{s}$ and $\delta_{\lambda}$ are defined as

$$
\delta_{r}=\left\{\begin{array}{ll}
1 & \text { for reactive transport } \\
0 & \text { for radionuclide transport }
\end{array} \quad \text { and } \delta_{\lambda}= \begin{cases}0 & \text { for reactive transport } \\
1 & \text { for radionuclide transport }\end{cases}\right.
$$


The first three terms on the left-hand side of (1) describe diffusion in the mobile pore water [Skagius and Neretnieks, 1988] through the immobile thin film in the immediate vicinity of the PM grains [de Marsily, 1986], and surface diffusion [Jahnke and Radke, 1987; Skagius and Neretnieks, 1988; Cook, 1989; Berry and Bond, 1992], respectively. The fourth term on the left-hand side (1) describes advective transport. The terms on the righthand side of equation (1) describe the dissolved species accumulation and radioactive decay in the pore water, in the immobile fraction, and on the PM grains due to sorption. Chemical reactions in the water phase are also accounted for [Cho, 1971]. A detailed discussion of these terms can be found in Moridis [1999], from where

$$
D_{m}=\phi\left(S-S_{r}\right)\left(\tau_{p} D_{0}+\alpha_{L} V\right) \quad \text { and } \quad D_{i}=\tau_{i} \phi S_{r} D_{0}
$$

where $D_{0}$ is the molecular diffusion coefficient of the dissolved species in water $\left[L^{2} T^{-1}\right]$, $\alpha_{L}$ is the longitudinal dispersivity $[L], \tau_{p}$ is the tortuosity factor of the pore paths [dimensionless], and $\tau_{i}$ is the tortuosity factor in the diffusion paths through the immobile fraction [dimensionless]. If surface diffusion cannot beneglected [Jensen and Radke, 1988], $D_{F}$ is given by [Jahnke, 1986; Jahnke and Radke, 1987]

$$
D_{F}=\tau_{s}(1-\phi) \rho D_{s}
$$

where $\tau_{s}$ is the tortuosity coefficient of the surface path [dimensionless], and $D_{s}$ is the surface diffusion coefficient $\left[L^{2} T^{-1}\right]$. For homogeneous PM systems there is theoretical justification [Cook, 1989] for the relationship $\tau_{s}=\frac{2}{3} \tau_{p}$.

The species concentration in the mobile and immobile water fractions are related through the linear equilibrium relationship [de Marsily, 1986],

$$
C_{i}=K_{i} C, \quad \mathcal{R}_{i}=K_{i} \mathcal{R}
$$

where $K_{i}$ is a dimensionless mass transfer coefficient. Equation (1) then becomes

$$
\begin{aligned}
D_{T} \frac{\partial^{2} C}{\partial x^{2}} & +D_{F} \frac{\partial^{2} F}{\partial x^{2}}-U \frac{\partial C}{\partial x} \\
& =\phi h\left(\frac{\partial C}{\partial t}+\delta_{\lambda} \lambda C\right)+(1-\phi) \rho\left(\frac{\partial F}{\partial t}+\delta_{\lambda} \lambda F\right)+\delta_{r} \phi h \frac{\partial \mathcal{R}}{\partial t}
\end{aligned}
$$


where

$$
D_{T}=\phi\left\{D_{0}\left[\tau_{p}\left(S-S_{r}\right)+\tau_{i} S_{r} K_{i}\right]+\left(S-S_{r}\right) \alpha_{L} V\right\}
$$

and

$$
h=\left(S-S_{r}\right)+S_{r} K_{i}
$$

\subsection{The Equations of Solute Sorption and First-Order Chemical Reaction}

Consideringthat sorption occurs as thedissolvedspecies diffusesthrough the immobile water fraction, and assuming linear equilibrium (LE) sorption, the following relationship applies:

$$
F_{p}=K_{d} K_{i} C
$$

where $K_{d}$ is the distribution coefficient $\left[M^{-1} L^{3}\right]$.

Linear kinetic physical (LKP) and linear irreversible physical (LIP) sorption are described by the equation [Moridis, 1999]

$$
\frac{\partial F_{p}}{\partial t}+\lambda F_{p}=k_{p}\left(K_{d} K_{i} C-\delta_{p} F_{p}\right)
$$

where $k_{p}$ is the kinetic constant of linear adsorption $\left[T^{-1}\right]$, and

$$
\delta_{p}= \begin{cases}1 & \text { for LKP sorption; } \\ 0 & \text { for linear LIP sorption }\end{cases}
$$

In the case of LIP sorption, $K_{d}$ does not represent the distribution coefficient of LE sorption, but is rather a proportionality factor.

Thefirst-order reversiblechemicalsorptionisrepresentedbythelinearkineticchemical (LKC) model

$$
\frac{\partial F_{c}}{\partial t}+\lambda F_{c}=k_{c}^{+} K_{i} C-k_{c}^{-} F_{c}
$$

where $k_{c}^{+}\left[M^{-1} L^{3} T^{-1}\right]$ and $k_{c}^{-}\left[T^{-1}\right]$ are the forward and backward kinetic constants, respectively. Note that equation (11) can be used in conjunction with the physical sorption 
equations to describe combined sorption [Cameron and Klute, 1977], e.g., physical and chemical sorption. Combined sorption accounts for the different rates at which a species is sorbed onto different PM constituents. Thus, sorption onto organic components may be instantaneous(LE), whilesorptionontomineralsurfacesmaybemuchslowerandkinetically controlled [Cameron and Klute, 1977].

The equations of a series of $N_{c}$ first-order chemical reaction are given by [Cho, 1971]

$$
\begin{gathered}
\frac{\partial \mathcal{R}_{1}}{\partial t}=\mathcal{K}_{1} C_{1}, \\
\frac{\partial \mathcal{R}_{2}}{\partial t}=\mathcal{K}_{2} C_{2}-\mathcal{K}_{1} C_{1}, \\
\vdots \\
\frac{\partial \mathcal{R}_{N_{c}}}{\partial t}=\mathcal{K}_{N_{c}} C_{N_{c}}-\mathcal{K}_{N_{c}-1} C_{N_{c}-1},
\end{gathered}
$$

where $\mathcal{K}_{j}\left(j=1, \ldots, N_{c}\right)$ is the chemical reaction rate constant $\left[T^{-1}\right]$, and $N_{c}$ is the number of chemical reactions in the series.

\subsection{The Solute Transport ODE in the Laplace Space}

2.3.1. Parent or Stable Species. After incorporating the sorption terms, the Laplace transform (LT) of the solute transport equation (5) yields the following Ordinary Differential Equation (ODE)

$$
D \frac{d^{2} \widehat{C}}{d x^{2}}-U \frac{d \widehat{C}}{d x}-E \widehat{C}=0
$$

where $\widehat{C}=\mathcal{L}\{C\}, \mathcal{L}\{\}$ denotes the $\mathrm{LT}$ of the quantity in the brackets,

$$
R= \begin{cases}h+w \psi & \text { for LE sorption; } \\ h+u \psi & \text { for LKP or LIP sorption, } \\ h+v \psi & \text { for LKC sorption, } \\ h+(w+u) \psi & \text { for combined LE and LKP/LIP sorption, } \\ h+(w+v) \psi & \text { for combined LE and LKC sorption } \\ h+(u+v) \psi & \text { for combined LKP/LIP and LKC sorption, }\end{cases}
$$




$$
D= \begin{cases}D_{T}+\phi \tau_{s} w \psi D_{s} & \text { for LE sorption; } \\ D_{T}+\phi \tau_{s} u \psi D_{s} & \text { for LKP or LIP sorption, } \\ D_{T}+\phi \tau_{s} v \psi D_{s} & \text { for LKC sorption, } \\ D_{T}+\phi \tau_{s}(w+u) \psi D_{s} & \text { for combined LE and LKP/LIP sorption, } \\ D_{T}+\phi \tau_{s}(w+v) \psi D_{s} & \text { for combined LE and LKC sorption, } \\ D_{T}+\phi \tau_{s}(u+v) \psi D_{s} & \text { for combined LKP/LIP and LKC sorption, } \\ w=K_{d} K_{i}, \quad u=\frac{k_{p} K_{d} K_{i}}{s+\lambda+\delta_{p} k_{p}}, \quad v=\frac{k_{c}^{+} K_{i}}{s+\lambda+k_{c}^{-}}, \quad \psi=\frac{(1-\phi)}{\phi} \rho\end{cases}
$$

and $s$ is the Laplace space parameter. The term $R$ is an expanded retardation factor, which can account for kinetic behavior [Moridis, 1999]. Its development involves the LT of the sorption from equations (8) through (11). It is straightforward to show that [Moridis, 1998]

$$
\widehat{F}=p \widehat{C}
$$

where $\widehat{F}=\mathcal{L}\{F\}$ and

$$
p= \begin{cases}w & \text { for LE sorption; } \\ u & \text { for LKP or LIP sorption, } \\ v & \text { for LKC sorption, } \\ w+u & \text { for combined LE and LKP/LIP sorption, } \\ w+v & \text { for combined LE and LKC sorption, } \\ u+v & \text { for combined LKP/LIP and LKC sorption. }\end{cases}
$$

Equation (13), subject to equations (14) through (19), is the Laplace space equation of solute transport in its most general form. Implicit in (13) are the assumptions that (a) $C(x, t=0)=0$, (b) $F(x, t=0)=0$, (c) $\mathcal{R}(x, t=0)=0$, and (d) in combined sorption, different sites are involved in each of the constituent types of sorption.

2.3.2. Daughter Species of Radioactive Decay. If the species is radioactive, the right-hand side of equation (5) is augmented by the term

$$
-\lambda_{\nu-1} m_{r}\left[\phi h C_{\nu-1}+(1-\phi) \rho F_{\nu-1}\right], \quad \text { where } \quad m_{r}=\frac{\mathcal{M}_{\nu}}{\mathcal{M}_{\nu-1}}
$$


$\mathcal{M}_{\nu}$ is the molecular weight of the $\nu$-th daughter $\left(1<\nu \leq N_{d}, N_{d}\right.$ being the total number of radioactive decay or reaction products), and $\nu-1$ refers to the decaying parent. Then, the Laplace space transport equation for any daughter product $\nu$ of the decay chain following a LE isotherm is given by

$$
D_{\nu} \frac{d^{2} \widehat{C}_{\nu}}{d x^{2}}-U \frac{d \widehat{C}_{\nu}}{d x}-E_{\nu} \widehat{C}_{\nu}=-G_{\nu} \widehat{C}_{\nu-1}
$$

where

$$
G_{\nu}=\phi m_{r} \lambda_{\nu-1} R_{\nu-1}
$$

If the daughter sorption is kinetically controlled, equations (9) and (11) need to account for the generation of daughter mass due to the decay of the sorbed parent, and become

$$
\frac{\partial F_{\nu}}{\partial t}+\lambda_{\nu} F_{\nu}-\lambda_{\nu-1} m_{r} \zeta_{\nu} F_{\nu-1}=k_{\alpha} C_{\nu}-k_{\beta} F_{\nu}
$$

where $F_{\nu-1}$ is the sorbed mass of the parent,

$$
k_{\alpha}=\left\{\begin{array}{ll}
k_{p} K_{d} K_{i} & \text { for LKP/LIP sorption, } \\
k_{c}^{+} K_{i} & \text { for LKC sorption, }
\end{array} \quad k_{\beta}= \begin{cases}k_{p} \delta_{p} & \text { for LKP/LIP sorption, } \\
k_{c}^{-} & \text {for LKC sorption, }\end{cases}\right.
$$

and $\zeta_{\nu}$ is the fraction of the mass of the decayed sorbed parent that remains sorbed as a daughter $\left(0 \leq \zeta_{\nu} \leq 1\right)$. The term $\zeta_{\nu}$ is introduced to account for the possibility that daughters can be ejected from grain surfaces due to recoil, e.g., the ejection of ${ }^{234} \mathrm{Th}$ from grain surfaces during the alpha decay of ${ }^{238} \mathrm{U}$ [Faure, 1977]. The LT of (22) returns

$$
\widehat{F}_{\nu}=p \widehat{C}_{\nu}+m_{r} p_{r} \widehat{C}_{\nu-1}
$$

where $p$ is obtained from equation (19), and

$$
p_{r}= \begin{cases}\frac{\lambda_{\nu-1} \zeta_{\nu} u}{s+\lambda_{\nu}+k_{p} \delta_{p}} & \text { for (a) LKP/LIP or (b) combined LE-LKP/LIP sorption } \\ \frac{\lambda_{\nu-1} \zeta_{\nu} v}{s+\lambda_{\nu}+k_{c}^{-}} & \text {for (a) LKC sorption or (b) combined LE-LKC sorption }\end{cases}
$$

For combined LKC and LKP/LIP sorption, $p_{r}$ is the sum of the two components in (24). Using (23) and (24), it is easy to show that equation (20) applies, but with

$$
G_{\nu}=\phi m_{r}\left[\lambda_{\nu-1} R_{\nu-1}-\left(s+\lambda_{\nu}\right) p_{r}\right]
$$


All other terms in (20) remain unchanged. Equations (20) through (25) are valid in any layer $n$. For a complete daughter ejection [Faure, 1977], $\zeta_{\nu}=0, p_{r}=0$, and (21) and (25) become identical.

2.3.3. Products of Chemical Reactions. If the species is a product of the $\nu$-th firstorder chemical reaction in the reaction chain (12), the right-hand side of equation (5) is augmented by the term $-\phi h \mathcal{K}_{\nu-1} C_{\nu-1}$. Then, equation (20) applies unchanged, but with

$$
G_{\nu-1}=\phi h \mathcal{K}_{\nu-1}
$$

\section{Transport in Layered Fractured Media}

The development of the equations for transport in a layered fractured media expands on the analysis of Tang et al. [1981] and Sudicky and Frind [1982]. A schematic of the fracture-matrix system is shown in Figure 1, in which the $N$ layers have different properties.

\subsection{Transport in the Matrix}

3.1.1. The ODE of Parent or Stable Species Transport in the Matrix. Advection in the matrix is neglected, that is $U_{n}^{m}=0$. Then the Laplace space ODE of the species transport in the matrix layer $n$ is given by

$$
D_{n}^{m} \frac{d^{2} \widehat{C}_{n}^{m}}{d x_{n}^{2}}-E_{n}^{m} \widehat{C}_{n}^{m}=0
$$

where the superscript $m$ denotes the matrix. The diffusive flux across the fracture-matrix interface is given by

$$
q_{n}=-\left.r_{n} D_{n}^{m} \frac{\partial C_{n}^{m}}{\partial x_{n}}\right|_{x_{n}=0}
$$

and differs from the analogous expression of Tang et al. [1981] in the inclusion of the active interface area reduction factor $r_{n}$. The term $r_{n}\left(1 \geq r_{n}>0\right)$ is defined as the ratio of the average interface area between mobile water in a fracture and its surrounding matrix to the average interface area between a fracture and the surrounding matrix. A detailed discussion on the subject can be found in Liu et al. [1998]. For a fully saturated fracture, $r_{n}=1$. 
3.1.2. The ODE of Daughter Transport in the Matrix. From equation (20), the Laplace space ODE of transport of the daughter $\nu$ in the matrix of layer $n$ is given by

$$
D_{n, \nu}^{m} \frac{d^{2} \widehat{C}_{n, \nu}^{m}}{d x_{n}^{2}}-E_{n, \nu}^{m} \widehat{C}_{n, \nu}^{m}=-G_{\nu}^{m} \widehat{C}_{n, \nu-1}^{m}
$$

where the term $G_{\nu}^{m}$ is computed from (21) to (26). The diffusive flux of the daughter $\nu$ across the fracture-matrix interface is given by equation (28).

\subsection{Transport in the Fractures}

3.2.1. Adjustments to Concepts and Equations. In fracture transport, the Darcy velocity $U_{n}$ in any layer $n$ is computed from the basic mass balance equation as

$$
U_{n}=\frac{Q_{w}}{M_{n} b_{n}}
$$

where $Q_{w}$ is the water influx rate per unit fracture thickness (in the $y$ direction, not shown in Figure 1) at the $z_{1}=0$ boundary $\left[L^{2} T^{-1}\right]$, and $2 b_{n}$ is the fracture aperture $[L]$. The parameter $M_{n}[L / L]$ is the relative fracture density, and is determined from the number of fractures in an arbitrary length $L_{x}$ (see Figure 1). The term $L_{x}$ is related to the matrix block half-width $X_{n}[L]$ and $b_{n}$ (see Figures $2 \mathrm{a}$ and $2 \mathrm{~b}$ ) through the relationship

$$
M_{n}=\frac{L_{x}}{2\left(X_{n}+b_{n}\right)}, \quad n=1, \ldots, N
$$

There are two different ways to treat the fractures. If the fractures are open, we have surface-based rather than volume-based sorption in the fractures of any layer $n$ $(n=1, \ldots, N)$. The following changes are then made:

(a) $F$ is now the mass of solute adsorbed per unit surface of the fracture $\left[M L^{-2}\right]$.

(b) From the mass balance equations, the term $(1-\phi) \rho$ in (17) is replaced by $1 / b_{n}$, where $b_{n}$ is the fracture half-width or half-aperture $[L]$ in layer $n$.

(c) The distribution coefficient of the fracture $K_{d}^{f}$ is now defined as the mass of solute adsorbed per unit area of surface divided by the concentration of solute in solution [Tang et al., 1981], with units [L]. 
(d) The kinetic constants $k_{c}^{+}$of chemical sorption in (11) have units $\left[L T^{-1}\right] ; k_{c}^{-}$in (11) have units $\left[M L^{-2} T^{-1}\right]$.

If the fractures are filled (a rather common occurrence), they are treated as a porous medium. Then, there is no need for the conceptual or mathematical adjustments in (1) through(4). Inbothopenandfilledfractures, theright-handsideofequation(5)isaugmented by the term

$$
Q_{n}=f_{n}^{q} q_{n}, \quad \text { where } f_{n}^{q}= \begin{cases}1 / b_{n} & \text { for open fractures } \\ 1 & \text { for filled fractures }\end{cases}
$$

and $q_{n}$ is described by (28).

3.2.2. The ODE of Parent or Stable Species Transport in the Fractures. The Laplacespaceequation forfracture transport alongthe $z$-coordinate(Figure 1)then becomes

$$
D_{n}^{f} \frac{d^{2} \widehat{C}_{n}^{f}}{d z_{n}^{2}}-U_{n} \frac{d \widehat{C}_{n}^{f}}{d z_{n}}-E_{n}^{f} \widehat{C}_{n}^{f}=\widehat{Q}_{n}
$$

where the $f$ superscript denotes the fracture, the $n$ subscripts denotes the layer, and $\widehat{Q}_{n}=\mathcal{L}\left\{Q_{n}\right\}$. Equation (31) is written in terms of the local coordinate $z_{n}$ in each layer $n$.

3.2.3. The ODE of Daughter Transport in the Fractures. The Laplace space ODE of transport for the daughter $\nu$ in the matrix of layer $n$ is given by

$$
D_{n, \nu}^{f} \frac{d^{2} \widehat{C}_{n, \nu}^{f}}{d z_{n}^{2}}-U_{n} \frac{d \widehat{C}_{n, \nu}^{f}}{d z_{n}}-E_{n, \nu}^{f} \widehat{C}_{n, \nu}^{f}=\widehat{Q}_{n}-G_{n}^{f} \widehat{C}_{n, \nu-1}^{f}
$$

All the terms in (32) are as previously defined.

\subsection{Initial and Boundary Conditions}

The initial and boundary conditions corresponding to the fracture equation are

$$
\begin{aligned}
& C_{n}^{f}\left(z_{n}, t=0\right)=0, \\
& C_{1}^{f}\left(z_{1}=0, t\right)=C_{z 0}(t), \\
& C_{n}^{f}\left(z_{n}=Z_{n}, t\right)=C_{n+1}^{f}\left(z_{n+1}=0, t\right), \quad n=1, \ldots, N-1, \\
& C_{N}^{f}\left(z_{N} \rightarrow \infty, t\right)=0,
\end{aligned}
$$


where $Z_{n}$ denotes the thickness of the $n$-th segment (layer). The time dependence of $C_{z 0}$ allows investigation of systems with time-variable upper boundaries. Some of the more common forms of $C_{z 0}(t)$ are

$$
C_{z 0}(t)= \begin{cases}C_{0} & \text { constant concentration } \\ C_{0} \exp \left[-\lambda\left(t+t_{d}\right)\right] & \text { decaying radionuclide concentration } \\ \sum_{i=1}^{N^{*}} C_{i}^{*}\left[\mathcal{U}\left(t-t_{i-1}^{*}\right)-\mathcal{U}\left(t-t_{i}^{*}\right)\right] & \text { variable pulse concentration }\end{cases}
$$

where $C_{0}$ is a constant, $t_{d}$ is the release delay (the time between radionuclide generation or storage, and the beginning of release), $\mathcal{U}\left(t-t^{*}\right)$ denotes the unit step function at time $t^{*}$, and $N^{*}$ is the number of the different pulses with concentration $C_{i}^{*}$. Note that $t_{0}^{*}=0$ and that, for $N^{*}=1$, we obtain the unit pulse of duration $t_{1}^{*}$.

The initial and boundary conditions corresponding to the matrix equation are

$$
\begin{aligned}
& C_{n}^{m}(x, t=0)=0, \\
& C_{n}^{m}(x=0, t)=C_{n}^{f}\left(z_{n}, t\right), \\
& \left\{\begin{array}{cc}
\frac{\partial C_{n}^{m}}{\partial x}(x=X, t)=0 & \text { for Case 1 (Figure 2a), } \\
C_{n}^{m}(x \rightarrow \infty, t)=0 & \text { for Case 2 (Figure 2b), }
\end{array}\right.
\end{aligned}
$$

where $X$ is the half-width of the matrix block (Figure 2). Case 1 in Figure 2a describes a finite system with a Neuman-type boundary at $x=X$. If dry fractures (i.e., fractures in which the water phase is discontinuous) occur in the rock matrix of Case 1, the half-width $X$ is replaced by $X^{*}=2 X /\left(n_{d}+1\right)$, where $n_{d}$ is the number of dry fractures evenly spaced along $x$ inthe matrix block(Figure 2b). Case 2 in Figure $2 b$ describes asemi-infinitesystem. The Laplace transforms of equations (33) through (35) are trivial. 


\section{The Laplace Space Equations}

\subsection{General Matrix Solutions in Each Layer}

4.1.1. Parent or Stable Species. Omitting for simplicity the $n$ subscript, and expanding on Tang et al. [1981] and Sudicky and Frind [1982], the solutions to (27) are

$$
\widehat{C}^{m}= \begin{cases}H^{c} \cosh [\theta(X-x)] & \text { for Case } 1 \\ H^{e} \exp (-\theta x) & \text { for Case } 2\end{cases}
$$

respectively, where $H^{c}$ and $H^{e}$ are parameters to be determined, and

$$
\theta=\theta(s)=\sqrt{\frac{E^{m}}{D^{m}}}
$$

From (36) and the Laplace transform of (35),

$$
\widehat{C}^{m}(x=0)= \begin{cases}H^{c} \cosh (\theta X)=\widehat{C}^{f} \Rightarrow H^{c}=\frac{\widehat{C}^{f}}{\cosh (\theta X)} & \text { for Case 1 } \\ H^{e}=\widehat{C}^{f} & \text { for Case 2 }\end{cases}
$$

from which

$$
\widehat{C}^{m}=\widehat{C}^{m}(x, s)= \begin{cases}\frac{\cosh [\theta(X-x)]}{\cosh (\theta X)} \widehat{C}^{f} & \text { for Case 1 } \\ \exp (-\theta x) \widehat{C}^{f} & \text { for Case 2 }\end{cases}
$$

The equations in (39) are applicable in any layer $n(n=1, \ldots, N)$.

4.1.2. Daughter or Reaction Products. Following the same approach, it is straightforward to show that the Laplace space solution of the ODE in (32) for any daughter or reaction product $\nu$ is given by

$$
\widehat{C}_{\nu}^{m}= \begin{cases}H_{\nu}^{c} \cosh \left[\theta_{\nu}(X-x)\right]+\sum_{\kappa=\nu-1}^{1}\left(\prod_{i=\nu}^{\kappa} A_{i \kappa}^{m}\right) H_{\kappa}^{c} \cosh \left[\theta_{\kappa}(X-x)\right] & \text { for Case 1 } \\ H_{\nu}^{e} \exp \left(-\theta_{\nu} x\right)+\sum_{\kappa=\nu-1}^{1}\left(\prod_{i=\nu}^{\kappa} A_{i \kappa}^{m}\right) H_{\kappa}^{c} \exp \left(-\theta_{\kappa} x\right) & \text { for Case 2 }\end{cases}
$$

where

$$
A_{i \kappa}^{m}=-\frac{G_{i}^{m}}{D_{i}^{m} \theta_{\kappa}^{2}-E_{i}^{m}} .
$$


The coefficients $H_{\nu}$ are given by the general expression

$$
H_{\nu}=\sum_{\kappa=1}^{\nu} T_{\nu, \kappa} \widehat{C}_{\kappa}^{f}
$$

where $T_{\nu, \kappa}$ are appropriate coefficients. Expressions for $H_{\nu}$ and $T_{\nu, \kappa}$ (the derivation of which is tedious but straightforward) are provided in Appendix A. Equation (40) shows that the solution of the matrix transport equation of the daughter or reaction product $\nu$ requires knowledge of the fracture solutions of all previous members of the decay or reaction chain.

\subsection{General Fracture Solutions in Each Layer}

4.2.1. Parent or Stable Species. From the Laplace transform of the diffusive flux in (30), and omitting for simplicity the subscript $n$,

$$
\widehat{Q}=\gamma f^{q} \widehat{C}^{f}
$$

where

$$
\gamma= \begin{cases}r D^{m} \theta \tanh (\theta X) & \text { for Case 1 } \\ r D^{m} \theta & \text { for Case 2 }\end{cases}
$$

Substituting in (31) and collecting terms,

$$
D^{f} \frac{d^{2} \widehat{C}^{f}}{d z^{2}}-U \frac{d \widehat{C}^{f}}{d z}-E^{*} \widehat{C}^{f}=0
$$

where $E^{*}=E^{f}+\gamma f^{q}$. The general solution to (45) is given by

$$
\widehat{C}^{f}=\widehat{C}^{f}(x, s)=\alpha \exp \left(\eta^{+} z\right)+\beta \exp \left(\eta^{-} z\right)
$$

where $\alpha$ and $\beta$ are parameters to be determined, and

$$
\eta^{ \pm}=\frac{U \pm \sqrt{U^{2}+4 D^{f} E^{*}}}{2 D^{f}}
$$

Equations (43)-(47) apply in any layer $n$.

4.2.2. Daughter or Reaction Products. From equations (30)-(32) and (40)-(42), for a daughter $\nu$

$$
\widehat{Q}_{\nu}=f^{q} r D_{\nu}^{m} W_{\nu}=f^{\prime} \sum_{\kappa=1}^{\nu} \gamma_{\nu, \kappa} \widehat{C}_{\kappa}^{f}
$$


Equation (48) is general and applies to both Case 1 and Case 2. Expressions for $W_{\nu}$ and $\gamma_{\nu, \kappa}$ are provided in Appendix B.

Substituting in (32) and collecting terms,

$$
D_{\nu}^{f} \frac{d^{2} \widehat{C}_{\nu}^{f}}{d z^{2}}-U \frac{d \widehat{C}_{\nu}^{f}}{d z}-E_{\nu}^{*} \widehat{C}_{\nu}^{f}=-G_{\nu} \widehat{C}_{\nu-1}^{f}+f^{q} \sum_{\kappa=1}^{\nu-1} \gamma_{\nu, \kappa} \widehat{C}_{\kappa}^{f}
$$

where $E_{\nu}^{*}=E_{\nu}^{f}+\gamma_{\nu, \nu} f^{q}$.

Following the same approach, it is straightforward to show that the Laplace space solution of any daughter or reaction product $\nu$ is given by

$$
\widehat{C}_{\nu}^{f}=\alpha_{\nu} \exp \left(\eta_{\nu}^{+} z\right)+\beta_{\nu} \exp \left(\eta_{\nu}^{-} z\right)+Y_{\nu}
$$

where

$$
Y_{\nu}=\sum_{\kappa=\nu-1}^{1} A_{\nu, \kappa}^{+} \alpha_{\kappa} \exp \left(\eta_{\kappa}^{+} z\right)+\sum_{\kappa=\nu-1}^{1} A_{\nu, \kappa}^{-} \beta_{\kappa} \exp \left(\eta_{\kappa}^{-} z\right)
$$

and

$$
A_{\nu, \kappa}^{ \pm}=\frac{B_{\nu, \kappa}^{ \pm}}{D_{\nu}^{f}\left(\eta_{\kappa}^{ \pm}\right)^{2}-U \eta_{\kappa}^{ \pm}-E_{\nu}^{*}}
$$

Expressions for $B_{\nu, \kappa}^{ \pm}$and for $\nu \leq 5$ are given in Appendix C. Equations (50) and (51) show that the solution of the fracture transport equation of the daughter or reaction product $\nu$ requires knowledge of all previous $\alpha_{\nu}$ and $\beta_{\nu}$, i.e., the solutions of all previous members of the chain.

\section{The Solution Approach}

\subsection{Determination of the $\alpha$ and $\beta$ Parameters}

Equation (46) defines a total of $2 N$ unknowns, i.e., the $\alpha$ and $\beta$ parameters in each of the $N$ subdomains. These are obtained from the solution of the following equations.

5.1.1. Boundary Equations. These apply to the $z_{1}=0$ point in the first layer $(n=1)$. From (46) and the Laplace transform of (33), for a known boundary concentration we have

$$
\alpha_{1}+\beta_{1}=\widehat{C}_{z 0}
$$


while for known flux boundary conditions

$$
\alpha_{1}\left(U_{1}-D_{1}^{f} \eta^{+}\right)+\beta_{1}\left(U_{1}-D_{1}^{f} \eta^{-}\right)=U_{1} \widehat{C}_{z 0}
$$

where $\widehat{C}_{z 0}=\mathcal{L}\left\{C_{z 0}\right\}$. For the common boundary conditions in (34),

$$
\widehat{C}_{z 0}= \begin{cases}\frac{C_{0}}{s} & \text { constant concentration } \\ \frac{C_{0} \exp \left(-\lambda t_{d}\right)}{s+\lambda} & \text { decaying radionuclide concentration } \\ \sum_{i=1}^{N^{*}} \frac{C_{i}^{*}}{s}\left[\exp \left(-s t_{i-1}^{*}\right)-\exp \left(-s t_{i}^{*}\right)\right] & \text { piecewise constant concentration. }\end{cases}
$$

For the limiting case of a system consisting of a single semi-infinite layer (i.e., $N=1$ ) with an open fracture and a constant concentration at $z_{1}=0, \alpha_{1}=0, \beta_{1}=C_{0} / \mathrm{s}$, and equation (46) is reduced to the Laplace space solutions obtained by Tang et al. [1981] (Case 2) and Sudicky and Frind [1982] (Case 1).

5.1.2. Concentration Equations. At the layer interfaces we have the equations

$$
\alpha_{n-1} \exp \left(\eta_{n-1}^{+} Z_{n-1}\right)+\beta_{n-1} \exp \left(\eta_{n-1}^{-} Z_{n-1}\right)-\alpha_{n}-\beta_{n}=0
$$

for $n=2, \ldots, N$. An additional equation is provided by the requirement that $\widehat{C}_{n}^{f}$ be finite for $Z_{N} \rightarrow \infty$, which dictates that $\alpha_{N}=0$.

5.1.3. Flux Equations. The remaining $N-1$ equations are provided by the equality of fluxes across the layer boundaries in the fractures, which dictates that

$$
M_{n-1} b_{n-1}\left[U_{n-1} C_{n-1}^{f}-D_{n-1}^{f} \frac{d C_{n-1}^{f}}{d z_{n-1}}\right]_{Z_{n-1}}=M_{n} b_{n}\left[U_{n} C_{n}^{f}-D_{n}^{f} \frac{d C_{n}^{f}}{d z_{n}}\right]_{0}
$$

in which the quantity in the brackets is computed at the value of the local $z$ coordinate indicated by the bracket subscript. From (46) and (57) we obtain

$$
\begin{aligned}
& \alpha_{n-1}\left[M_{n-1} b_{n-1}\left(U_{n-1}-D_{n-1}^{f} \eta_{n-1}^{+}\right)\right] \exp \left(\eta_{n-1}^{+} Z_{n-1}\right) \\
& +\beta_{n-1}\left[M_{n-1} b_{n-1}\left(U_{n-1}-D_{n-1}^{f} \eta_{n-1}^{-}\right)\right] \exp \left(\eta_{n-1}^{-} Z_{n-1}\right) \\
& \quad-\alpha_{n}\left[M_{n} b_{n}\left(U_{n}-D_{n}^{f} \eta_{n}^{+}\right)\right]-\beta_{n}\left[M_{n} b_{n}\left(U_{n}-D_{n}^{f} \eta_{n}^{-}\right)\right]=0
\end{aligned}
$$


where $n=1, \ldots, N-1$.

5.1.4. Equations for Daughters. For a daughter product $\nu$ of radioactive decay or reaction, the following changes are made to equations (53) through (58):

(a) In the right-hand side of equations (53) and (54), the term $\widehat{C}_{z 0}$ is replaced by $\widehat{C}_{\nu, z 0}$, where $\widehat{C}_{\nu, z 0}=\mathcal{L}\left\{C_{\nu, z 0}\right\}$, and $C_{\nu, z 0}$ is the concentration of daughter $\nu$ at $z_{1}=0$. For a constant $C_{\nu, z 0}, \widehat{C}_{\nu, z 0}$ can be obtained from equation (55). For a $z_{1}=0$ boundary with a decaying radionuclide concentration, $\widehat{C}_{\nu, z 0}$ is computed from the Laplace transform of the mass balance equation $\frac{\partial C_{\nu, z 0}}{\partial t}=\lambda_{\nu} C_{\nu, z 0}-\lambda_{\nu-1} C_{\nu-1, z 0}$ as

$$
\widehat{C}_{\nu, z 0}=\frac{C_{\nu, z 0}}{s+\lambda_{\nu}} \exp \left(-\lambda t_{d}\right)+m_{r} \frac{\lambda_{\nu-1}}{s+\lambda_{\nu}} \exp \left(-\lambda t_{d}\right) \widehat{C}_{\nu-1, z 0} .
$$

For a reaction chain, equation (59) indicates a recursive reaction.

(b) The zero on the right-hand side of the layer interface equation (56) is replaced by $Y_{\nu, n}\left(z_{n}=0\right)-Y_{\nu, n-1}\left(z_{n-1}=Z_{n}\right)$ for $n=2, \ldots, N$.

(c) Equation (57) applies unchanged. The zero on the right-hand side of equation (58) is replaced by the known quantity

$$
\begin{aligned}
M_{n} b_{n}\left[U_{n} Y_{\nu, n}\right. & \left.-D_{\nu, n}^{f} \frac{d Y_{\nu, n}}{d z_{n}}\right]_{0} \\
& -M_{n-1} b_{n-1}\left[U_{n-1} Y_{\nu, n-1}-D_{\nu, n-1}^{f} \frac{d Y_{\nu, n-1}}{d z_{n-1}}\right]_{Z_{n-1}}
\end{aligned}
$$

\subsection{The Laplace Space Solutions}

The generality and complexity of these equations preclude the development of closedform solutions for $\alpha_{i}, \beta_{i}(i=1, \ldots, N)$. Consequently, it is not possible to analytically invert equations (46) or (50), and to obtain a closed-form equation for concentration in time. The problem is alleviated by numerically inverting the Laplace space solutions. The algebraic equations discussed in Section 5.1 may be written in a general matrix form as:

$$
\mathbf{M} \overrightarrow{\mathbf{X}}=\overrightarrow{\mathbf{B}},
$$


where $\mathbf{M}$ is the coefficient matrix, $\overrightarrow{\mathbf{X}}$ is the vector of the unknowns, and $\overrightarrow{\mathbf{B}}$ is the composite vector of knowns. Solution of (71) returns the vector

$$
\overrightarrow{\mathbf{X}}=\left(\begin{array}{c}
\overrightarrow{\mathbf{X}}_{1} \\
\overrightarrow{\mathbf{X}}_{2} \\
\vdots \\
\overrightarrow{\mathbf{X}}_{N}
\end{array}\right), \quad \text { where } \quad \overrightarrow{\mathbf{X}}_{i}=\left(\begin{array}{c}
\alpha_{i} \\
\beta_{i}
\end{array}\right), i=1, \ldots, N
$$

The solution of the matrix equation (60) necessitates arithmetic values for the $s$ parameter of the Laplace space. These are provided by the numerical inversion scheme of DeHoog et al. [1982] that uses complex values for $s$. The quantities $\mathbf{M}, \overrightarrow{\mathbf{X}}$ and $\overrightarrow{\mathbf{B}}$ assume the complex type of $s$. A detailed discussion of the application of this method and its performance can be found in Sudicky [1990] and Moridis [1998].

The $\alpha_{i}$ and $\beta_{i}$ computed from the matrix equation (60) are then used to obtain all the $\widehat{C}_{n}^{f}$ solutions $(i=1, \ldots, N)$. The corresponding $\widehat{C}_{n}^{m}$ solutions are obtained from $\widehat{C}_{n}^{f}$ and equations (39) or (40)-(42). Note that the solutions for daughters or reaction products require knowledge of the solutions of all the previous members in the chain.

\subsection{Numerical Inversions of the Laplace Space Solutions}

The various time-variable concentrations can be determined by numerically inverting the Laplace space solutions, i.e.,

$$
C_{n}^{f}(x, t)=\mathcal{L}^{-1}\left\{\widehat{C}_{n}^{f}(x, s)\right\}, \quad C_{n}^{m}(x, t)=\mathcal{L}^{-1}\left\{\widehat{C}_{n}^{m}(x, s)\right\}
$$

where $\mathcal{L}^{-1}\{\}$ denotes the inverse Laplace transform of the quantity in the brackets. Details on the inversion will not be discussed here; they can be found in DeHoog et al. [1982].

\section{Treatment of Special Conditions}

\subsection{Misaligned Fractures}

The analysis presented thus far assumes that the effect of fracture offset on transport is negligible. This may not be the case for large fracture spacing or at short observation times. 
The process that accounts for fracture misalignment is described in Figure 3. The increased travel path of the transporting water caused by the offset fractures is indicated by the horizontal pathway at the confluence of the $n$ and $n+1$ layers in Figure 3a, and its effect is described by the addition of an "interlayer", i.e., a pseudo-layer (Figure 3b) with the following characteristics:

(a) A thickness $Z_{I}=\max \left\{X_{n}, X_{n+1}\right\}$ if $M_{n}>M_{n=1}$, or $Z_{I}=\min \left\{X_{n}, X_{n+1}\right\}$ if $M_{n}<M_{n+1}$.

(b) A relative frequency $M_{I}=M_{n}$.

(c) An open or filled fracture of half-width $b_{I}$, through which water flows between the $n$ and $n+1$ layers. The properties of the fracture in the interlayer are independent of those in the layers above and below.

(d) A complex matrix, composed of the matrices of both the $n$ and $n+1$ layers. In Figure $3 \mathrm{~b}$, the matrices of the $n$ and $n+1$ layers are positioned on the left and right sides of the fracture, respectively. The two components of the matrix are assumed to be semi-infinite, as illustrated by their rotation by $90^{\circ}$ (with respect to the original layer orientation) in Figure 3b. Then, the flux into the composite matrix of the interlayer is computed from equation (43), but with $\gamma \equiv \gamma_{I}$, where

$$
\gamma_{I}=\frac{1}{2}\left(\gamma_{n}+\gamma_{n+1}\right)
$$

and $\gamma_{n}, \gamma_{n+1}$ are computed from equation (44).

Thus, considerationof misaligned fracturestransformsasystem of $N$ layerstoasystem of $N+N_{I}$ layers, where $N_{I}$ is the number of interlayers. The solution of the augmented system does not pose any particular challenges and proceeds in the manner discussed in Section 5. Note that this approximation involves the longest possible travel path and the largest possible amount of tracer diffusion. This is because diffusion into the matrix of the interlayer (see Figure $3 \mathrm{~b}$ ) is larger than that into the $n$ and $n+1$ layers (along the layer interface) owing to steeper gradients and their semi-infinite nature. Thus, the assumption of fracture alignment provides the most conservative solution, while the assumption of fracture 
misalignment (as described by the concept of interlayers) reflects the least conservative scenario. These two solutions provide the limits that bracket the true solution.

\subsection{Occasional Unfractured Layers}

If the layered system includes unfractured (porous) layers (e.g., Layer 3 in Figure 1), these are treated as a combination of a pseudo-matrix (representing the nonflowing portion of the layer) and a pseudo-fracture representing the flowing portion of the layer. In essence, unfractured layers are treated as filled-fracture systems, and all the equations apply unchanged. The properties of the unfractured medium are assigned to both the pseudomatrix and the pseudo-fracture. The relative sizes of $b$ and $X$ can describe the flowing and non-flowing portions of the porous medium. If water flows uniformly through the porous medium, $X=0$. This approach maintains water mass and flux balance.

It is obvious that, for unfractured media, $L_{x}=2\left(b_{n}+X_{n}\right)$, i.e., $M_{n}=1$. Note that water saturations $S$ must be obtained from the solution of the steady-state flow equation because the derivation of the transport equations is based on time-invariant flow conditions and cannot compute changes in $S$.

\subsection{Transport in Layered Unfractured Media}

This is a limiting case of the scenario discussed in Section 6.2. Setting the non-flowing portion of the matrix $X_{n}=0(n=1, \ldots, N)$ transforms the problem into that of 1-D solute transport in a layered porous (unfractured) system. Then, all the solutions derived here apply unchanged.

\section{Verification}

A FORTRAN program was written to obtain the semianalytical (SA) solutions developed in Sections 4 through 6 by first solving (60), and then performing the numerical inversion indicated in (62). This code, named FRACL, accounts for all the processes, 
phenomena and conditions discussed in Sections 2 through 6. It can obtain solutions for a system involving an arbitrary number of layers $N$ of any combination of porous and/or fractured media, and up to 4 daughters. It is computationally very efficient, and required less than 10 seconds for any of the problems discussed in Sections 7 or 8 .

FRACL is verified through comparisons to analytical solutions of radioactive solute and colloid transport in 1-D porous (unfractured) media and quasi-2-D fractured media. In all cases, FRACL solutions are first obtained in a system consisting of a single semi-infinite layer (i.e., $N=1$ ). The domain is then subdivided into three layers in the $z$ direction, and FRACL solutions for this multilayered system $(N=3)$ are obtained. Coincidence of the analytical solutions to the FRACL solutions for $N=1$ and for $N=3$ verifies FRACL.

\subsection{Tests FS1 and FS2: Radioactive Solute Transport in Fractures}

Tests FS1 and FS2 describe transport with LE sorption in the fracture-matrix system of Case 1 (parallel fractures, Figures 2a) and Case 2 (single fracture, Figure 2b), respectively. The corresponding analytical solutions were developed by Sudicky and Frind [1982] and Tang et al. [1981]. The values of the parameters used for the computation of the analytical and the SAsolutions areas in Sudickyand Frind [1982], and are listed in Table 1. A constant concentration (CC) condition is applied at $z_{1}=0$.

Figure 4 shows the distribution of the relative concentration $C_{R}$ (defined as $C_{R}=$ $\left.C_{n}^{f} / C_{z 0}\right)$ in the fractures along the $z$ axis at (a) $t=1,000$ days in Test FS1 and (b) $t=10,000$ days in Test FS2. In both tests, the analytical solution and the two FRACL solutions (for $N=1$ and $N=3$ ) are identical in the first 5 significant digits.

\subsection{Tests PS1 to PS4: Radioactive Solute Transport in Unfractured Porous Media}

Tests PSI to PS4 are designed to confirm the ability of the SA solutions to describe transport in unfractured media without any modification. The solution to this problem is provided by Bear [1979], and accounts for LE sorption and radioactive decay. 
The values of the parameters used for the computation of the analytical and the SA solutions of Tests PS1 to PS4 are listed in Table 2. In all four tests, a constant concentration condition is applied at $z_{1}=0$. The solute is a nondecaying isotope in Tests PS1 and PS2, and a decaying radionuclide in Tests PS3 and PS4. LE sorption is considered in Tests PS2 and PS3, but is ignored in Tests PS1 and PS4.

Figure 5 shows the distribution of the relative concentration $C_{R}$ along the $z$ axis at $t=200$ days. The SA predictions of $C_{R}$ distributions for both $N=1$ and $N=3$ are identical with the analytical solutions of Bear [1979].

\subsection{Test PS5: Transport of a Three-Member Radioactive Solute Chain in Unfractured Porous Media}

This test is designed to verify the ability of the SA solutions to describe the transport of reactive chains in unfractured media without any modification. An analytical solution to this problem was developed by Harada et al. [1980], and accounts for LE sorption, radioactive decay, and time-variable boundary conditions.

Test PS5 describes the transport of the radioactive chain ${ }^{234} \mathrm{U} \rightarrow{ }^{230} \mathrm{Th} \rightarrow{ }^{226} \mathrm{Ra}$ through a sorbing porous medium. The concentration of ${ }^{234} \mathrm{U}$ (i.e., the parent radionuclide) at the $z_{1}=0$ is not constant over time, but subject to radioactive decay. The initial concentrations of the ${ }^{230} \mathrm{Th}$ and ${ }^{226} \mathrm{Ra}$ daughter radionuclides at the $z_{1}=0$ boundary are zero, but increase over time because of the decay of their parents.

The values of the parameters used for the computation of the analytical and the SA solutions of Test PS5 are as in Harada et al. [1980], and are listed in Table 3. Figure 6shows that the analytical solutions at $t=10,000$ years coincide with the SA predictions (for both $N=1$ and $N=3$ ) of the $C_{R}$ distributions of the three radioactive chain members.

\section{Analysis and Test Problems}

In this section the transport of various radionuclides is studied in layered systems 
(involving both fractured and porous layers) of different characteristics and properties. The $D_{0}$ and $\lambda$ of the radionuclides discussed here appear in Table 4.

\subsection{Problem 1: Importance of Fracture Misalignment}

This problem studies the importance of fracture misalignment on transport, as quantified by the concept of interlayers (discussed in Section 6.1). The following analysis focuses on the effects of the presence of such interlayers, in conjunction with other parameters of the hydrogeologic layers and of the species. The flow velocity in all cases of Problem 1 was $U=0.1 \mathrm{~m} /$ day, the system was saturated $(S=1)$, and the $z=0$ boundary was kept at a constant concentration $\left(C_{R}=1\right)$.

8.1.1. Case 1-a: Effect of fracture offset (interlayers). This case involves the transport of the nonsorbing solute species ${ }^{3} \mathrm{H}$ in a layered fractured system with fracture offsets and various interlayer characteristics. Case 1-a involves three sub-cases: 1-a1, 1-a2 and 1-a3. The geometry of the reference Case 1-a1 of the layered fractured system is described in Table 5, while the hydraulic properties of the fractured layers are shown in Table 6. The three main layers (identified as Layers \# 1,3 and 5 in Table 6) were fractured media (FM), while the interlayers (identified as Layers \# 2 and 4) were considered to be fracture interlayers (FI, i.e., horizontal open fractures connecting the vertical fractures in the layers above and below).

The characteristics of Cases 1-a2 and 1-a3 are explained in Table 7, which shows only the differences from the base Case 1-a1. Thus, Cases 1-a2 and 1-a3 differ from Case 1-a1 in that the interlayers are porous interlayers (PI), i.e., the horizontal features connecting the fractured layers are either fractures filled with porous media or unfractured porous media. Flow and transport occurs through a porous medium with different transport behavior than in the FIsof Case 1-a1. The hydraulic properties of the porous media in the PIsin Cases 1-a2 and $1-\mathrm{a} 3$ are the same as those of the porous matrix in the overlaying and underlying layers. The connecting PI in Cases $1-\mathrm{a} 2$ and $1-\mathrm{a} 3$ have $\mathrm{a}=0.025 \mathrm{~m}$ and $0.1 \mathrm{~m}$, respectively. Note that in PI and PM layers there are no fractures and $b$ represents the half-width of the flowing 
portion of the matrix.

The results of the three subcases of Case 1-a are shown in Figure 7, which shows the fracture $C_{R}$. The presence of the interlayers in Figure 7 is marked by the vertical steps in the $C_{R}$ profiles (caused by the fact that Figure 7 indicates the vertical coordinate $\mathrm{z}$ and not the length of the travel path. For the nonsorbing ${ }^{3} \mathrm{H}$ and at early times, the retardation caused by the presence of the FI is measurable, as compared to the case with aligned fractures (no interlayer, denoted by NI in Figure 7-included for comparison). This was expected because of the longer travel path in the case of FIs, which increase the amount of ${ }^{3} \mathrm{H}$ diffusing into the porous matrix and result in lower fracture concentrations. At the same early times, the retardation caused by the PIs can be substantial and increases with the half-width $b$ of the PI. These results also conform with expectations because of the slower flow velocities in the porous media of the PI (as compared to those in the fractures of the FIs), which increase the residence time and diffusion into the porous matrix.

Figure 7 also shows that the effect of the interlayers keeps decreasing with time. This was expected in Case 1-a because the travel path increase caused by the interlayers is small (as the layer half-width $X$ is only $0.25 \mathrm{~m}$ ) and ${ }^{3} \mathrm{H}$ is nonsorbing (leaving diffusion into the matrix as the only mechanism removing the radionuclide from the flowing water). At $t=10^{4}$ days, the presence of interlayers of any kind (FI vs. PI) has no effect on the concentration profile in the fractures.

\subsubsection{Case 1-b: Combined effect of interlayers and matrix width of the fractured} layers. This case involves three subcases: 1-b1, 1-b2 and 1-b3 (see Table 7). Cases 1-b1, $1-\mathrm{b} 2$ and 1-b3 differed from Cases $1-\mathrm{a} 1,1-\mathrm{a} 2$ and $1-\mathrm{a} 3$ in that $X=2.5 \mathrm{~m}$ instead of 0.25 $\mathrm{m}$, thus substantially increasing the travel path and residence time of ${ }^{3} \mathrm{H}$ in the interlayers. This is expected to increase retardation, especially at early times.

Figure 8 confirms this expectation. At $t=10^{2}$ days, the presence of the relatively fast flowing FI is sufficient to reduce $C_{R}$ in the fracture by about four orders of magnitude. The effect is more pronounced in Case 1-b3 (PI with $b=0.1 \mathrm{~m}$ ). The same pattern is observed 
at $t=10^{3}$ days, at which time the retardation in Case $1-\mathrm{b} 3$ remains very substantial. This is caused by the reduction of the advective and dispersive components of transport (because velocity decreases as $b$ increases) in addition to the reduction of the molecular diffusion component (due to the smaller $\phi$ and $\tau$ values in the filled fracture, see equation (2)). Remarkably, stronger retardation is observed in Case 1-b1 (FI) than in case 1-b2 (PI with $b=0.025 \mathrm{~m}$ ). This is attributed to the larger solute mass in the PI, which is less affected by diffusion into the matrix (about the same in both cases). As in Case 1-a, the effect of the fracture offset (presence of interlayers) decreases with time.

The conclusion reached from these results is that the effect of fracture offsets (interlayers) increases with the matrix block size of the fractured layers. This is consistent with expectations because the travel path increases substantially in fractured system with large $X$, with a corresponding increase in residence time and diffusion into the matrix.

\subsubsection{Case 1-c: Combined effect of interlayers and water saturation $S$ of the} fractured layers. This case involved two subcases: 1-c1 and 1-c2 (see Table 7). Cases 1-cl differed from Case 1 -al in that $S^{m}=0.8$ and $S^{f}=0.5$ instead of $S^{m}=S^{f}=1$. Cases 1-c2 differed from Case 1-a3 in that $S^{m}=S^{f}=0.8$ instead of $S^{m}=S^{f}=1$. The effect of $S$ is exhibited through its impact on the water velocity: a higher pore velocity $V$ is needed to maintain the same $U$ if $S$ decreases. Thus, faster transport was expected in this case, with a corresponding decrease in the importance of the increased travel path caused by the fracture offset.

The results in Figure 9 confirm these expectations. Transport is faster than in Cases $1-a$ and 1-b, while the importance of the fracture offset (presence of interlayers) decreases in systems with the same water mass flow rate but with decreasing $S_{w}$.

\subsection{Problem 2: Radioactive Solute Transport in a Complex Multi-Layered System}

The complex geological system in Problem 2 is comprised of 14 layers and interlayers of fractured and porous media. The geometry and configuration of the system are described 
in Table 8, and the rock properties and conditions are listed in Table 9. Linear equilibrium sorptionis assumed, and thesorption coefficientsof thevariousradionuclides inthefractures and in the matrix of the various layers ( $K_{d}^{f}$ and $K_{d}^{m}$, respectively) are listed in Table 10. The water velocity $U$ at $z=0$ as in Problem 1 .

8.2.1. ${ }^{3} \mathrm{H}$ Transport. The fracture $C_{R}$ profiles of the nonsorbing ${ }^{3} \mathrm{H}$ for both constant concentration (CC) and decaying (radioactively) concentration (DC) at the $z=0$ boundary are shown in Figure 10, which includes observations at the following times: $t_{1}=10^{4}$ days, $t_{2}=5 \times 10^{4}$ days, $t_{3}=10^{5}$ days, $t_{4}=2.5 \times 10^{5}$ days and $t_{5}=5 \times 10^{5}$ days.

The various layers can be generally identified by a change in the $C_{R}$ slope, while the interlayers are indicated by vertical sections of the $C_{R}$ curves (as the abscissa is the $z$ coordinate rather than the travel path). For a $\mathrm{CC}$ boundary, the $C_{R}$ distribution reaches a steady state for $t \geq t_{4}$. As expected, the effect of the DC boundary is a $C_{R}$ profile that is progressively lower than the one for a CC boundary, never reaches steady state, and is outside the $C_{R}$ range $\left(<10^{-9}\right)$ for $t \geq t_{4}$.

8.2.2. ${ }^{99} \mathrm{Tc}$ Transport. ${ }^{99} \mathrm{Tc}$ (in its pertechnate $\mathrm{TcO}_{4}^{-}$speciation) is a non-sorbing radionuclide with a longer half life than ${ }^{3} \mathrm{H}$ (see Table 4). Two boundary conditions were considered in this case: a CC boundary and a piece-wise continuous (step) concentration (PC) boundary, i.e.,

$$
C_{R}(z=0)= \begin{cases}1 & \text { for } t \leq 5 \times 10^{4} \text { days } \\ 0 & \text { for } t>5 \times 10^{4} \text { days }\end{cases}
$$

The $C_{R}$ profiles in the fractures of the layered geologic system (at the same times as in the case of ${ }^{3} \mathrm{H}$ in Section 8.2.1) are shown in Figure 11. The effect of the longer half life is evident in the $C_{R}$ profile for $\mathrm{CC}$ boundary, which indicates that ${ }^{99} \mathrm{Tc}$ advances much further in the formation than ${ }^{3} \mathrm{H}$ at the same times (the difference is due to radioactive decay), and does not appear to have reached steady state at $t=t_{5}$. The change in the boundary concentration over time in the PC boundary case results in $C_{R}$ profiles that show aprogressivelylarger(withtime) ${ }^{99} \mathrm{Tc}$-freezoneneartheboundary, whilethe $C_{R}$ further into 
the formation keeps decreasing and deviating from that for constant boundary concentration (with which it coincides fully or in part for $t \leq t_{4}$ ).

As indicated in the case of ${ }^{3} \mathrm{H}$, the various layers and interlayers can be generally identified from changes in the $C_{R}$ slope. Transport in fast flowing fractures (e.g., in the case of narrow fractures with large matrix blocks under a layer of wider fractures and narrow matrix blocks) can also be identified by a near-horizontal portion of the $C_{R}$ profile.

8.2.3. ${ }^{237} \mathrm{~Np}$ Transport. The $C_{R}$ profile of the moderately sorbing ${ }^{237} \mathrm{~Np}$ for a $\mathrm{CC}$ boundary is shown in Figure 12. The observation times are: $t_{1}=5 \times 10^{4}$ days, $t_{2}=10^{5}$ days, $t_{3}=5 \times 10^{5}$ days, $t_{4}=10^{6}$ days, $t_{5}=2.5 \times 10^{6}$ days and $t_{6}=5 \times 10^{6}$ days.

The slower transport of ${ }^{237} \mathrm{~Np}$ (compared to that of ${ }^{99} \mathrm{Tc}$ ) is caused by sorption and, to a far lesser extent, by increased diffusion into the matrix. Despite its longer half-life, the transport of ${ }^{237} \mathrm{~Np}$ appears to be about an order of magnitude slower than that of ${ }^{99} \mathrm{Tc}$, and does not appear to have reached steady state at $t=t_{6}$.

The $C_{R}$ profiles along the $x$ axis in the matrices of the various layers at $t=t_{6}$ are shown in Figure 13. The different shape of the curves is a function of their location (with respect to the $z=0$ boundary and to the solute front) and of the transport properties of the matrix in the various layers.

\subsection{Problem 3: Solute Transport of a Three-Member Radioactive Decay Chain in a Complex Multi-Layered System}

Problem 3 describes the transport of the radioactive chain ${ }^{239} \mathrm{Pu} \rightarrow{ }^{235} \mathrm{U} \rightarrow{ }^{231} \mathrm{~Pa}$ through the complex multilayered system described in Problem 2 (Tables 8 and 9). The sorption coefficients $K_{d}^{f}$ and $K_{d}^{m}$ of the ${ }^{239} \mathrm{Pu}$ parent in the various layers are listed in Table 10. The sorption coefficients of ${ }^{235} \mathrm{U}$ and ${ }^{231} \mathrm{~Pa}$ in the fractures and in the matrix were assumed to be $5 \%$ and $50 \%$ of those for ${ }^{239} \mathrm{Pu}$, respectively. $C_{R}$ profiles of the three radionuclides were obtained at the following observation times: $t_{1}=10^{5}$ days, $t_{2}=10^{6}$ days, $t_{3}=10^{7}$ days, $t_{4}=10^{8}$ days, $t_{5}=10^{9}$ days, and $t_{6}=10^{10}$ days. Two boundary conditions were considered: a CC and a DC boundary. 
8.3.1. ${ }^{239} \mathrm{Pu}$ Transport. Figure 14 shows the $C_{R}$ profiles of ${ }^{239} \mathrm{Pu}$ in the fractures for constant boundary concentration and a decaying boundary concentration. There is no or little deviation of the two curves until $t=t_{3}$. The fracture $C_{R}$ in DC case at $t=t_{4}$ is substantially lower than that of the $\mathrm{CC}$ case, and the $C_{R}$ for a $\mathrm{DC}$ boundary is less than $10^{-9}$ for $t \geq t_{5}$

An interesting observation is that, for a $\mathrm{CC}$ boundary, the ${ }^{239} \mathrm{Pu}$ front does not advance deep into the formation despite observation times orders of magnitude larger than those for the ${ }^{237} \mathrm{~Np}$ transport. This is due to the very strong sorption of ${ }^{239} \mathrm{Pu}$ onto the matrix and fractures of the layers and, to a lesser extent, the shorter half life of ${ }^{239} \mathrm{Pu}$ (compared to that of ${ }^{237} \mathrm{~Np}$. Note that the $C_{R}$ profile appears to have reached steady state at $t \geq t_{5}$.

In addition to the transport of the members of the chain, the transport of ${ }^{239} \mathrm{Pu}$ was studied separately, assuming a CC boundary and a $r \leq 1$ (see Equation (38) and the corresponding discussion). This describes a situation in which not all the contact area between fracture and matrix contributes to transport (e.g., because of a partially dry fracture which constitutes a discontinuity in the water phase). In this case, $r=S_{w}^{f}$ in the fractured layers and interlayers (FM or FI), and $r=1$ elsewhere.

The effect of $r \leq 1$ in Figure 15 appears to have a substantial impact on transport, and results in a ${ }^{239} \mathrm{Pu}$ front that reaches much further (i.e., about three times deeper) in the geologic profile than that for $r=1$. This is a direct consequence of a reduced area for ${ }^{239} \mathrm{Pu}$ diffusion from the fractures into the matrix, which leaves a larger amount of ${ }^{239} \mathrm{Pu}$ in the fractures where advection is fast and sorption relatively small (compared to the matrix). Thus, the transport of strongly sorbing radionuclides in fractured systems may be substantially influenced (enhanced) by partially dry fractures.

8.3.2. ${ }^{235} \mathrm{U}$ Transport. The fracture $C_{R}$ profiles of ${ }^{235} \mathrm{U}$ for $\mathrm{CC}$ and DC boundaries and for $t \leq t_{4}$ are shown in Figure 16. The $C_{R}$ of the DC solution always exceeding that from the CC solution, and significantly so (as imposed) in the vicinity of $z=0$. A very significant observation is that, in either case, $C_{R} \simeq 1$ for $t \geq t_{4}$ in the top $120 \mathrm{~m}$ of the 
domain. This is even more the case in Figure 17, which shows the $C_{R}$ of ${ }^{235} U$ for $t \geq 4$ and gives a more detailed picture of the $C_{R}$ distribution near the value of 1 . The results in Figures 16 and 17, in conjunction with the observations from Figure 14, indicate that for $t \geq t_{4}$, practically all of the radionuclide that advances deep into the formation is the ${ }^{235} \mathrm{U}$ daughter. The transport of ${ }^{235} \mathrm{U}$ is faster, the front reaches deeper, and $C_{R} \simeq 1$ because ${ }^{235} \mathrm{U}$ is generally weaker sorbing than ${ }^{239} \mathrm{Pu}$ and it has an extremely long half life. The obvious implication is that studies of ${ }^{239} \mathrm{Pu}$ transport cannot neglect the transport of the ${ }^{235} \mathrm{U}$ daughter, which is the dominant radionuclide at longer times.

Note from Figure 17 that, for $t=t_{4}$ and a DC boundary, $C_{R}>1$, i.e., the ${ }^{235} \mathrm{U}$ concentration in the fractures exceeds the initial concentration of the ${ }^{239} \mathrm{Pu}$ parent at the $z=0$ boundary. This is possible because the boundary (which introduces a radionuclide mixture composed of all the members of the ${ }^{239} \mathrm{Pu}$ decay chain) is now contributing a stream ofalmost $100 \%{ }^{235} \mathrm{U}$, whichis addedtothe ${ }^{235}$ Uproduced fromthe(almost complete)decay of ${ }^{239} \mathrm{Pu}$ already in the fractures and matrix of the system. As expected, the $C_{R}$ from the CC solution at $t=t_{4}$ is lower than that from the DC solution (Figure 16). For $t>t_{4}$, the $\mathrm{CC}$ solutions exceed the DC solutions because the decay of the ${ }^{235} \mathrm{U}$ at the boundary is beginning to have an effect on the fracture distribution of $C_{R}$. This is particularly evident at $t=t_{6}$. Note that steady state is not reached (in either the CC or the DC boundary cases) even after $t_{6}=10^{10}$ days because of the extremely long half life of ${ }^{235} \mathrm{U}$.

8.3.3. ${ }^{231} \mathrm{~Pa}$ Transport. The fracture $C_{R}$ profiles of ${ }^{231} \mathrm{~Pa}$ for $\mathrm{CC}$ and DC boundaries are shown in Figure 18. The $C_{R}$ levels of ${ }^{231} \mathrm{~Pa}$ are quite low because of the very long half life of its ${ }^{235} \mathrm{U}$ parent, its own shorter half life, and its stronger tendency to sorb. The $C_{R}$ increases with time for both DC and CC boundaries. The CC profile has always lower concentrations because there are all derived solely from the decay of ${ }^{235} U$ (the boundary doesnotsupplyanyadditional ${ }^{231}$ PainaCCregime). Note thatineither case,concentrations reach a steady state at about $t=t_{4}$. 


\section{Summary}

In this paper, semianalytical solutions are developed for the problem of transport of radioactive or reactive solute tracers through a layered system of heterogeneous fractured media with misaligned fractures. The solutions allow any number and combination of fractured and/or porous layers that can vary in hydraulic and transport properties, fracture frequency, water saturation, fracture flow, and fracture-matrix interaction.

The tracer transport equations in the matrix account for (a) diffusion, (b) solute surface diffusion, (c) mass transfer between the mobile and immobile water fractions, (d) linear kinetic or equilibrium physical, chemical or combined solute sorption or colloid filtration, and (e) radioactive decay or first order chemical reactions. Any number of radioactive decay daughter products (or products of a linear, first-order reaction chain) can be tracked. The tracer transport equations in the fractures account for the same processes, as well as for advection and hydrodynamic dispersion. A wide array of boundary conditions (constant or time-variable, concentration or flux) can be accommodated.

Analytical solutions describing transport in the fracture and the matrix of each layer are first obtained in the Laplace space. These are impossible to invert analytically, and are numerically inverted by the method of DeHoog et al. [1982] to yield the solutions in

time. These SA solutions are verified against analytical solutions of limiting cases of solute transport in fractured media. Additional verification is provided by comparisons against analytical solutions of transport in porous (unfractured) media.

The SA solutions are then tested in a series of hypothetical problems of increasing complexity. The effectof important parameters on the transport of ${ }^{3} \mathrm{H},{ }^{237} \mathrm{~Np}$ and ${ }^{239} \mathrm{Pu}$ (and its daughters) is investigated in several test problems involving layered fractured geological systems. Fracture misalignment appears to significantly affect transport if water flow (and, consequently, transport) between the fractures of the overlaying and the underlying layers occurs through a porous connecting pathway.

The semianalytical solutions are computationally very efficient, requiring less than 10 
seconds of execution time for the examples studied in this paper. The results of the test problems indicate that the semianalytical solutions can easily solve the problem of transport of parent and daughter radioactive species in multilayered heterogeneous systems under a variety of boundary conditions. Thus, they can provide a simple and effective tool to predict radionuclide transport in subsurface environments involving saturated/unsaturated flow through variably fractured media (such as transport from the potential repository through the fractured rock layers in the UZ of Yucca Mountain to the water table). While such predictions are quasi 2-D and do not account for spatial variability and flow effects in the 3-D continuum of the subsurface (such as perched water bodies, flow diversion and flow focusing), they can provide bounding estimates that bracket the true solution.

Acknowledgments. ThisworkwassupportedbytheDirector, OfficeofCivilianRadioactive Waste Management, U.S. Department of Energy, through Memorandum Purchase Order EA9013MC5X between Bechtel SAIC Company, LLC and the Ernest Orlando Lawrence Berkeley National Laboratory (Berkeley Lab). The support is provided to Berkeley Lab through the U.S. Department of Energy Contract No. DE-AC03-76SF00098. The author is indebted to Gundmundur (Bo) Bodvarsson for providing the important context information that is discussed in the introduction. The careful review of Stefan Finsterle is gratefully acknowledged despite the considerable misery it caused the author. Chao Shan and John Apps are also thanked for their insightful review comments.

\section{Appendix A: The $H_{\nu}$ and $T_{\nu, \kappa}$ Coefficients}

For Case $2(X \rightarrow \infty)$, the $H_{\nu} \equiv H_{\nu}^{e}$ of the first five members of a radioactive or reactive chain $(\nu=1, \ldots, 5)$ are

$$
\begin{aligned}
& H_{1}^{e}=\widehat{C}_{1}^{f} \\
& H_{2}^{e}=\widehat{C}_{2}^{f}-A_{21} \widehat{C}_{1}^{f} \\
& H_{3}^{e}=\widehat{C}_{3}^{f}-A_{32} \widehat{C}_{2}^{f}+A_{21}\left(A_{32}-A_{31}\right) \widehat{C}_{1}^{f}
\end{aligned}
$$




$$
\begin{aligned}
& H_{4}^{e}=\widehat{C}_{4}^{f}-A_{43} \widehat{C}_{3}^{f}+A_{32}\left(A_{43}-A_{42}\right) \widehat{C}_{2}^{f} \\
&-A_{21}\left[A_{32}\left(A_{43}-A_{42}\right)-A_{31}\left(A_{43}-A_{41}\right)\right] \widehat{C}_{1}^{f} \\
& H_{5}^{e}=\widehat{C}_{5}^{f}-A_{54} \widehat{C}_{4}^{f}+A_{43}\left(A_{54}-A_{53}\right) \widehat{C}_{3}^{f} \\
&-A_{32}\left[A_{43}\left(A_{54}-A_{53}\right)-A_{42}\left(A_{54}-A_{52}\right)\right] \widehat{C}_{2}^{f} \\
& \quad+A_{21}\left\{A_{32}\left[A_{43}\left(A_{54}-A_{53}\right)-A_{42}\left(A_{54}-A_{52}\right)\right]\right. \\
&\left.\quad-A_{31}\left[A_{43}\left(A_{54}-A_{53}\right)-\dot{A}_{41}\left(A_{54}-A_{51}\right)\right]\right\} \widehat{C}_{1}^{f}
\end{aligned}
$$

in which the $m$ superscript of the $A$ factors (equation (52)) are omitted for simplicity. The terms $T_{\nu, \kappa}$ inequation (53)can beeasilyidentified byinspection. Byfollowing theemerging pattern, the development of the expressions for $H_{\nu}$ for $\nu>5$ is tedious but straightforward.

The $H_{\nu} \equiv H_{\nu}^{c}$ expressions (corresponding to Case 1) are entirely analogous, and are derived by dividing $H_{\nu}^{e}$ by $\cosh \left(\theta_{\nu} X\right)$. For example, for $\nu=2$,

$$
H_{2}^{c}=\frac{\widehat{C}_{2}^{f}}{\cosh \left(\theta_{2} X\right)}-\frac{A_{21} \widehat{C}_{1}^{f}}{\cosh \left(\theta_{2} X\right)}
$$

\section{Appendix B: The $W_{\nu}$ and $\gamma_{\nu, \kappa}$ Coefficients}

For Case $2(X \rightarrow \infty)$, the $W_{\nu} \equiv W_{\nu}^{e}$ of the first 5 members of a radioactive or reactive chain $(\nu=1, \ldots, 5)$ are

$$
\begin{aligned}
& W_{1}^{e}=\theta_{1} C_{1}^{f} \\
& W_{2}^{e}=\theta_{2} C_{2}^{f}+A_{21}\left(\theta_{1}-\theta_{2}\right) \widehat{C}_{1}^{f} \\
& W_{3}^{e}=\theta_{3} C_{3}^{f}+A_{32}\left(\theta_{2}-\theta_{3}\right) \widehat{C}_{2}^{f}+A_{21}\left[A_{31} \theta_{1}-A_{32} \theta_{2}+\left(A_{32}-A_{31}\right) \theta_{3}\right] \widehat{C}_{1}^{f} \\
& W_{4}^{e}=\theta_{4} C_{4}^{f}+A_{43}\left(\theta_{3}-\theta_{4}\right) \widehat{C}_{3}^{f}+A_{32}\left[A_{42} \theta_{2}-A_{43} \theta_{3}+\left(A_{43}-A_{42}\right) \theta_{4}\right] \widehat{C}_{2}^{f} \\
& +A_{21}\left\{A_{31} A_{41} \theta_{1}-A_{32} A_{42} \theta_{2}+A_{43}\left(A_{32}-A_{31}\right) \theta_{3}\right. \\
& \left.\quad-\left[A_{32}\left(A_{43}-A_{42}\right)-A_{31}\left(A_{43}-A_{41}\right)\right] \theta_{4}\right\} \widehat{C}_{1}^{f}
\end{aligned}
$$




$$
\begin{gathered}
W_{5}^{e}=\theta_{5} C_{5}^{f}+A_{54}\left(\theta_{4}-\theta_{5}\right) \widehat{C}_{4}^{f}+A_{43}\left[A_{53} \theta_{3}-A_{54} \theta_{4}+\left(A_{54}-A_{53}\right) \theta_{5}\right] \widehat{C}_{3}^{f} \\
+A_{32}\left\{A_{42} A_{52} \theta_{2}-A_{43} A_{53} \theta_{3}+A_{54}\left(A_{43}-A_{42}\right) \theta_{4}\right. \\
\left.\quad-\left[A_{43}\left(A_{54}-A_{53}\right)-A_{42}\left(A_{54}-A_{52}\right)\right] \theta_{5}\right\} \widehat{C}_{2}^{f} \\
+A_{21}\left\{A_{31} A_{41} A_{51} \theta_{1}-A_{32} A_{42} A_{52} \theta_{2}+A_{43} A_{53}\left(A_{32}-A_{31}\right) \theta_{3}\right. \\
-A_{54}\left[A_{32}\left(A_{43}-A_{42}\right)-A_{31}\left(A_{43}-A_{41}\right)\right] \theta_{4} \\
+\left[A_{32}\left[A_{43}\left(A_{54}-A_{53}\right)-A_{42}\left(A_{54}-A_{52}\right)\right]\right. \\
\left.\left.-A_{31}\left[A_{43}\left(A_{54}-A_{53}\right)-A_{41}\left(A_{54}-A_{51}\right)\right]\right] \theta_{5}\right\} \widehat{C}_{1}^{f}
\end{gathered}
$$

in which the $m$ superscript of the $A$ factors (equation (52)) are omitted for simplicity.

We obtain $W_{\nu}^{c}$ for Case 1 by replacing $\theta_{\nu}$ by $\theta_{\nu} \tanh \left(\theta_{\nu} X\right)$ in $W_{\nu}^{e}$. Thus, for $\nu=2$ and Case 1,

$$
W_{2}^{c}=\theta_{2} \tanh \left(\theta_{2} X\right) C_{2}^{f}+A_{21}\left[\theta_{1} \tanh \left(\theta_{1} X\right)-\theta_{2} \tanh \left(\theta_{2} X\right)\right] \widehat{C}_{1}^{f}
$$

The terms $\gamma_{\nu, \kappa}$ are easy to obtain from (59) and the $W_{\nu}^{c}, W_{\nu}^{e}$ expressions by inspection. Extension for $\nu>5$ follows the same pattern.

\section{Appendix C: The $B_{\nu, \kappa}^{ \pm}$Coefficients}

The $B_{\nu, \kappa}^{ \pm}$coefficients of up to the first 5 members of a radioactive or reactive chain $(\nu=1, \ldots, 5, \kappa=1, \ldots, \nu-1)$ are given by the following general expressions:

$$
\begin{gathered}
B_{\nu, \nu-1}^{ \pm}=\gamma_{\nu, \nu-1} f^{q}-G_{\nu}^{f} \\
B_{\nu, \nu-2}^{ \pm}=\left(\gamma_{\nu, \nu-1} f^{q}-G_{\nu}^{f}\right) A_{\nu-1, \nu-2}^{ \pm}+\gamma_{\nu, \nu-2} f^{q} \\
B_{\nu, \nu-3}^{ \pm}=\left(\gamma_{\nu, \nu-1} f^{q}-G_{\nu}^{f}\right) A_{\nu-1, \nu-3}^{ \pm}+\left(\gamma_{\nu, \nu-2} A_{\nu-2, \nu-3}^{ \pm}+\gamma_{\nu, \nu-3}\right) f^{q} \\
B_{\nu, \nu-4}^{ \pm}=\left(\gamma_{\nu, \nu-1} f^{q}-G_{\nu}^{f}\right) A_{\nu-1, \nu-4}^{ \pm}+\left(\gamma_{\nu, \nu-2} A_{\nu-2, \nu-4}^{ \pm}+\gamma_{\nu, \nu-3} A_{\nu-3, \nu-4}^{ \pm}+\gamma_{\nu, \nu-4}\right) f^{q}
\end{gathered}
$$

The coefficients $A^{ \pm}$needed for the computation of $B^{ \pm}$are obtained from equation (63). All other terms are as discussed in Section 5.2. Extension for $\nu>5$ follows the same pattern. 


\section{References}

Bandurraga, T.M., and G.S. Bodvarsson, Calibrating hydrogeologic parameters for the 3D site-scale unsaturated zone model of Yucca Mountain, Nevada, J. Contam. Hydrol., $38(1-3), 25-46,1999$.

Bear, J., Hydraulics of Groundwater, McGraw-Hill, New York, 1979.

Berry, J. A., and K. A. Bond, Studies of the extent of surface diffusion in the migration of radionuclides through geological media, Radiochimica Acta, 58/59, 329-335, 1992.

Cameron, D.R., and A. Klute, Convective-dispersive solute transport with a combined equilibrium and kinetic adsorption model, Water Resour. Res., 13(1), 183-188, 1977.

Cho, C.M., Convective transport of ammonium with nitrification in soil, Can. J. Soil Sci., $51,339-350,1971$.

Cook, A. J., A desk study of surface diffusion and mass transport in clay, Report WE/88/34, Commission of the European Communities, Directorate-General, Sciences Research and Development, Louxemburg, 1989.

DeHoog, F.R., J.H. Knight, and A.N. Stokes, An improved method for numerical inversion of Laplace transforms, SIAM J. Sci. Stat. Comput., 3(3), 357-366, 1982.

de Marsily, G., Quantitative Hydrogeology, Academic Press, San Diego, 1986.

Faure, G, Principles of Isotope Geology, John Wiley and Sons, New York, New York, 1977.

Harada, M., P.L Chambré, M. Foglia, K. Higashi, F. Iwamoto, D. Leung, D.H. Pigford, and D. Ting, Migration of radionuclides through sorbing media, Report LBL-10500, Lawrence Berkeley Laboratory, Berkeley, California, 1980.

Jahnke, F.M., Electrolyte diffusion in montmorillonite engineered barriers, Ph.D. dissertation, Univ. of Calif., Berkeley, 1986.

Jahnke, F.M., and C.J. Radke, Electrolyte diffusion in compacted montmorillonite engineered barriers, in Coupled Processes Associated With Nuclear Waste Repositories, pp. 287-297, Academic Press, Orlando, 1987.

Jensen, D.J., and C.J. Radke, Cation diffusion through compacted sodium montmorillonite 
at elevated temperature, J. Soil Sci., 39, 53-64, 1988.

Liu, H.H., C. Doughty, and G.S. Bodvarsson, An active-fracture formulation for modeling flow and transport in fractured rocks, Water Resour. Res., 34, (10), 2633-2646, 1998.

Montazer, P., andW.E. Wilson, Conceptualhydrologicmodelofflowintheunsaturatedzone, Yucca Mountain, Nevada, Resources Investigations Report 84-4355, U.S. Geological Survey, Denver, Colorado, 1984.

Moridis, G. J., Semianalytical solutions for parameter estimation in diffusion cell experiments, Water Resour. Res., 35(6), 1729-1740, 1999.

Moridis, G. J., A set of semianalytical solutions for parameter estimation in diffusion cell experiments, Report LBNL-41857, Lawrence Berkeley National Laboratory, Berkeley, Calif., 1998.

Moridis, G. J., Alternative formulations of the Laplace Transform Boundary Element (LTBE) numerical method for the solution of diffusion-type equations, in Boundary Element Technology VII, pp. 815-833, Computational Mechanics Publications, Boston, and Elsevier Applied Science, New York, 1992.

Robinson, N.I., J.M. Sharp, and I. Kreisel, Contaminant transport in sets of parellel finite fractures with fracture skins, J. Cont. Hydrol., 3l, (83-109, 1998.

Skagius, K., and I. Neretnieks, Measurements of cesium and strontium diffusion in biotite gneiss, Water Resour. Res., 24(1), 75-84, 1988.

Sudicky, E.A., The Laplace transform Galerkin technique for efficient time-continuous solution of solute transport in double-porosity media, Geoderma, 46209-232, 1990.

Sudicky, E.A., and E.O. Frind, Contaminant transport in fractured porous media: analytical solution for a two-member decay chain in a single fracture, Water Resour. Res., 20(7), $1021-1027,1984$.

Sudicky, E.A., and E.O. Frind, Contaminant transport in fractured porous media: analytical solutions for a system of parallel fractures, WaterResour. Res., I8(6), 1634-1642, 1982.

Tang, D.H., E.O. Frind, and E.A. Sudicky, Contaminant transport infractured porous media: 
Analytical solution for a single fracture, Water Resour. Res., 17(3), 555-564, 1981.

Table 1. Input parameters in Test FS1

\begin{tabular}{|c|c|}
\hline Parameters & Values \\
\hline Water saturation $S$ & 1 \\
\hline PM grain density $\rho$ & $2600 \mathrm{~kg} / \mathrm{m}^{3}$ \\
\hline$D_{0}$ & $1.6 \times 10^{-9} \mathrm{~m}^{2} / \mathrm{s}$ \\
\hline Fracture aperture $2 b$ & $10^{-4} \mathrm{~m}$ \\
\hline Fracture $S$ & 1 \\
\hline Fracture $\phi$ & 1 \\
\hline Fracture $\tau$ & 1 \\
\hline Fracture $K_{d}$ & $0 \mathrm{~m}$ \\
\hline Longitudinal dispersivity $\alpha_{L}$ in the fracture & $0.1 \mathrm{~m}$ \\
\hline Fracture flow velocity $V$ & $0.1 \mathrm{~m} / \mathrm{day}$ \\
\hline Matrix block width $2 X$ & $0.5 \mathrm{~m}$ \\
\hline Matrix $S$ & 1 \\
\hline Matrix $\phi$ & 0.01 \\
\hline Matrix $\tau$ & 0.1 \\
\hline Matrix $K_{d}$ & $0 \mathrm{~m} / \mathrm{kg}$ \\
\hline Radionuclide $T_{1 / 2}$ & 12.35 years (tritium) \\
\hline$Z_{1}, Z_{2}, Z_{3}$ (for $N=3$ ) & $1 \mathrm{~m}, 9 \mathrm{~m}, \infty$ \\
\hline
\end{tabular}


Table 2. Input parameters in Tests PS1 to PS4

\begin{tabular}{|c|c|}
\hline Parameters & Values \\
\hline$\rho$ & $2600 \mathrm{~kg} / \mathrm{m}^{3}$ \\
\hline$D_{0}$ & $5 \times 10^{-2} \mathrm{~m}^{2} /$ day \\
\hline$S$ & 1 \\
\hline$\phi$ & 0.1 \\
\hline$\tau$ & 1 \\
\hline$V$ & $0.1 \mathrm{~m} /$ day \\
\hline$K_{d}($ Tests PS1 and PS4) & $0 \mathrm{~m}^{3} / \mathrm{kg}$ \\
\hline$K_{d}$ (Tests PS2 and PS3) & $4.2735042 \times 10^{-5} \mathrm{~m}^{3} / \mathrm{kg}$ \\
\hline$T_{1 / 2}$ (Tests PS1 and PS2) & $\infty($ stable isotopes) \\
\hline$T_{1 / 2}$ (Tests PS3 and PS4) & 100 days \\
\hline$Z_{1}, Z_{2}, Z_{3}$ (for $\left.N=3\right)$ & $10 \mathrm{~m}, 10 \mathrm{~m}, \infty$ \\
\hline
\end{tabular}


Table 3. Input parameters in Test PS5

\begin{tabular}{|c|c|}
\hline Parameters & Values \\
\hline$\rho$ & $2600 \mathrm{~kg} / \mathrm{m}^{3}$ \\
\hline$D_{0}$ & $1000 \mathrm{~m}^{2} /$ year \\
\hline$S$ & 1 \\
\hline$\phi$ & 0.3 \\
\hline$\tau$ & 1 \\
\hline$V$ & $100 \mathrm{~m} /$ year \\
\hline$K_{d}$ for ${ }^{234} \mathrm{U}$ & $1.64819 \mathrm{~m}^{3} / \mathrm{kg}$ \\
\hline$K_{d}$ for ${ }^{230} \mathrm{Th}$ & $8.24159 \mathrm{~m}^{3} / \mathrm{kg}$ \\
\hline$K_{d}$ for ${ }^{226} \mathrm{Ra}$ & $8.22528 \times 10^{-2} \mathrm{~m}{ }^{3} / \mathrm{kg}$ \\
\hline$T_{1 / 2}$ of ${ }^{234} \mathrm{U}$ & $2.45 \times 10^{5}$ years \\
\hline$T_{1 / 2}$ of ${ }^{230} \mathrm{Th}$ & $7.54 \times 10^{4}$ years \\
\hline$T_{1 / 2}$ of ${ }^{226} \mathrm{Ra}$ & $1.60 \times 10^{3}$ years \\
\hline$Z_{1}, Z_{2}, Z_{3}($ for $N=3)$ & $50 \mathrm{~m}, 150 \mathrm{~m}, \infty$ \\
\hline
\end{tabular}


Table 4. Radionuclide properties used in the transport simulations of Section 8

\begin{tabular}{|c|c|c|}
\hline Radionuclide & $D_{0}\left(\mathrm{~m}^{2} / \mathrm{s}\right)$ & $\lambda=\frac{\ln 2}{T_{1 / 2}}(1 / \mathrm{s})$ \\
\hline${ }^{3} \mathrm{H}$ & $1.60 \times 10^{-9}$ & $1.778 \times 10^{-9}$ \\
\hline${ }^{99} \mathrm{Tc}$ & $4.55 \times 10^{-10}$ & $1.031 \times 10^{-13}$ \\
\hline${ }^{237} \mathrm{~Np}$ & $7.12 \times 10^{-10}$ & $1.026 \times 10^{-14}$ \\
\hline${ }^{239} \mathrm{Pu}$ & $6.08 \times 10^{-10}$ & $9.114 \times 10^{-13}$ \\
\hline${ }^{235} \mathrm{U}$ & $6.08 \times 10^{-10}$ & $3.1023 \times 10^{-17}$ \\
\hline${ }^{231} \mathrm{~Pa}$ & $6.08 \times 10^{-10}$ & $6.7583 \times 10^{-13}$ \\
\hline
\end{tabular}

Table 5. Layer geometry in Case 1-a of Problem 1

\begin{tabular}{|c|c|c|c|}
\hline Layer\# & Type & Parameter & Value \\
\hline 1 & FM & $Z$ & $5 \mathrm{~m}$ \\
& & $X$ & $0.25 \mathrm{~m}$ \\
& & $b$ & $5 \times 10^{-5} \mathrm{~m}$ \\
\hline 2 & FI & $b$ & $5 \times 10^{-5} \mathrm{~m}$ \\
\hline 3 & FM & $Z$ & $10 \mathrm{~m}$ \\
& & $X$ & $0.25 \mathrm{~m}$ \\
& & $b$ & $5 \times 10^{-5} \mathrm{~m}$ \\
\hline 4 & FI & $b$ & $5 \times 10^{-5} \mathrm{~m}$ \\
\hline 5 & FM & $Z$ & $\infty \mathrm{m}$ \\
& & $X$ & $0.25 \mathrm{~m}$ \\
& & $b$ & $5 \times 10^{-5} \mathrm{~m}$ \\
\hline
\end{tabular}


Table 6. Properties in Case 1-a1 of Problem 1

\begin{tabular}{|c|c|c|}
\hline Layer \# & Parameters & Values \\
\hline $1,3,5$ & $\begin{array}{c}\alpha_{L} \\
\phi^{m} \\
\tau^{m}=\tau_{p}^{m}=\tau_{i}^{m}=\tau_{s}^{m} \\
\phi^{f} \\
\tau^{f}=\tau_{p}^{f}=\tau_{i}^{f}=\tau_{s}^{f} \\
K_{i}^{m}=K_{i}^{f} \\
S_{w}^{m}=S_{w}^{f}\end{array}$ & $\begin{array}{c}0.1 \mathrm{~m} \\
0.01 \\
0.1 \\
1 \\
1 \\
1 \\
1\end{array}$ \\
\hline 2,4 & $\begin{array}{c}\alpha_{L} \\
\phi^{m} \\
\tau^{m}=\tau_{p}^{m}=\tau_{i}^{m}=\tau_{s}^{m} \\
\phi^{f} \\
\tau^{f}=\tau_{p}^{f}=\tau_{i}^{f}=\tau_{s}^{f} \\
K_{i}^{m}=K_{i}^{f} \\
S^{m}=S^{f}\end{array}$ & $\begin{array}{c}0.1 \mathrm{~m} \\
0.01 \\
0.1 \\
1 \\
1 \\
1 \\
1\end{array}$ \\
\hline
\end{tabular}


Table 7. Parameter variations in the various cases of Problem 1

\begin{tabular}{|c|c|c|c|c|}
\hline Case \# & Layer \# & Type & Parameter & Value \\
\hline $1-a 2$ & 2,4 & PI & $b$ & $0.025 \mathrm{~m}$ \\
\hline $1-\mathrm{a} 3$ & 2,4 & PI & $b$ & $0.10 \mathrm{~m}$ \\
\hline \multirow[t]{2}{*}{$1-b 1$} & $1,3,5$ & $\mathrm{FI}$ & $X$ & $2.5 \mathrm{~m}$ \\
\hline & 2,4 & FI & $Z$ & $2.5 \mathrm{~m}$ \\
\hline \multirow[t]{3}{*}{$1-b 2$} & $1,3,5$ & FI & $X$ & $2.5 \mathrm{~m}$ \\
\hline & 2,4 & PI & $Z$ & $2.5 \mathrm{~m}$ \\
\hline & & & $b$ & $0.025 \mathrm{~m}$ \\
\hline \multirow[t]{3}{*}{$1-b 3$} & $1,3,5$ & $\mathrm{FI}$ & $X$ & $2.5 \mathrm{~m}$ \\
\hline & 2,4 & PI & $Z$ & $2.5 \mathrm{~m}$ \\
\hline & & & $b$ & $0.10 \mathrm{~m}$ \\
\hline \multirow[t]{2}{*}{$1-c 1$} & All & FM, FI & $S_{w}^{m}$ & 0.8 \\
\hline & & & $S_{w}^{f}$ & 0.5 \\
\hline \multirow[t]{2}{*}{$1-c 2$} & All & FM, PI & $S_{w}^{m}=S_{w}^{f}$ & 0.8 \\
\hline & 2,4 & PI & $b$ & $0.1 \mathrm{~m}$ \\
\hline
\end{tabular}


Table 8. Layer geometry in Problem 2

\begin{tabular}{|c|c|c|c|c|}
\hline Layer \# & Type & $Z(\mathrm{~m})$ & $X(\mathrm{~m})$ & $b(\mathrm{~m})$ \\
\hline 1 & FM & 10 & 0.5 & $10^{-4}$ \\
\hline 2 & PI & & & $5 \times 10^{-2}$ \\
\hline 3 & FM & 10 & 0.25 & $5 \times 10^{-5}$ \\
\hline 4 & PI & & & $2.5 \times 10^{-2}$ \\
\hline 5 & FM & 10 & 3 & $2 \times 10^{-4}$ \\
\hline 6 & PM & 5 & & \\
\hline 7 & FM & 15 & 0.1 & $2 \times 10^{-4}$ \\
\hline 8 & PI & & & $10^{-1}$ \\
\hline 9 & FM & 10 & 4 & $2 \times 10^{-5}$ \\
\hline 10 & $\mathrm{FI}$ & & & $2 \times 10^{-5}$ \\
\hline 11 & FM & 20 & 1 & $5 \times 10^{-5}$ \\
\hline 12 & PM & 5 & & \\
\hline 13 & FM & 30 & 6 & $8 \times 10^{-5}$ \\
\hline 14 & PM & $\infty$ & & \\
\hline
\end{tabular}


Table 9. Rock properties in Problem 2

\begin{tabular}{|c|c|c|c|c|c|c|}
\hline Layer \# & $\phi^{m}$ & $\tau^{m}$ & $S_{w}^{m}$ & $\phi^{f}$ & $\tau^{f}$ & $S_{w}^{f}$ \\
\hline 1 & 0.15 & 0.5 & 0.7 & 1 & 1 & 0.2 \\
\hline 2 & 0.3 & 0.3 & 1 & 0.3 & 0.3 & 0.4 \\
\hline 3 & 0.1 & 0.4 & 0.6 & 1 & 1 & 0.15 \\
\hline 4 & 0.35 & 0.3 & 1 & 0.35 & 0.3 & 0.3 \\
\hline 5 & 0.05 & 0.5 & 0.8 & 1 & 1 & 0.1 \\
\hline 6 & 0.35 & 0.8 & 0.9 & 0.35 & 0.8 & 0.9 \\
\hline 7 & 0.025 & 0.2 & 0.9 & 1 & 1 & 0.1 \\
\hline 8 & 0.2 & 0.3 & 0.9 & 0.2 & 0.3 & 0.4 \\
\hline 9 & 0.01 & 0.2 & 0.95 & 1 & 1 & 0.05 \\
\hline 10 & 0.01 & 0.2 & 0.95 & 1 & 1 & 0.05 \\
\hline 11 & 0.05 & 0.15 & 0.95 & 1 & 1 & 0.05 \\
\hline 12 & 0.1 & 0.1 & 0.9 & 0.2 & 0.1 & 0.9 \\
\hline 13 & 0.05 & 0.1 & 1 & 1 & 1 & 1 \\
\hline 14 & 0.1 & 0.1 & 1 & 0.1 & 0.1 & 1 \\
\hline
\end{tabular}


Table 10. Transfer coefficients in Problem 2

\begin{tabular}{|c|c|c|c|c|c|c|}
\hline \multirow{2}{*}{ Layer } & \multicolumn{2}{|c|}{${ }^{3} \mathrm{H}$ or ${ }^{99} \mathrm{Tc}$} & \multicolumn{2}{|c|}{${ }^{237} \mathrm{~Np}$} & \multicolumn{2}{c|}{${ }^{239} \mathrm{Pu}$} \\
\cline { 2 - 7 } & $K_{d}^{m}\left(^{*}\right)$ & $K_{d}^{f}(\dagger)$ & $K_{d}^{m}\left(^{*}\right)$ & $K_{d}^{f}(\dagger)$ & $K_{d}^{m}\left(^{*}\right)$ & $K_{d}^{f}(\dagger)$ \\
\hline 1 & 0 & 0 & $6 \times 10^{-4}$ & $3 \times 10^{-8}$ & $6 \times 10^{-2}$ & $3 \times 10^{-6}$ \\
\hline 2 & 0 & 0 & $8 \times 10^{-4}$ & $8 \times 10^{-4}$ & $8 \times 10^{-2}$ & $8 \times 10^{-2}$ \\
\hline 3 & 0 & 0 & $7 \times 10^{-4}$ & $3.5 \times 10^{-8}$ & $7 \times 10^{-2}$ & $3.5 \times 10^{-6}$ \\
\hline 4 & 0 & 0 & $8 \times 10^{-4}$ & $8 \times 10^{-4}$ & $8 \times 10^{-2}$ & $8 \times 10^{-2}$ \\
\hline 5 & 0 & 0 & $8 \times 10^{-4}$ & $4 \times 10^{-8}$ & $8 \times 10^{-2}$ & $4 \times 10^{-6}$ \\
\hline 6 & 0 & 0 & $10^{-4}$ & $10^{-4}$ & $10^{-2}$ & $10^{-2}$ \\
\hline 7 & 0 & 0 & $10^{-3}$ & $5 \times 10^{-7}$ & $10^{-1}$ & $5 \times 10^{-5}$ \\
\hline 8 & 0 & 0 & $8 \times 10^{-4}$ & $8 \times 10^{-4}$ & $8 \times 10^{-2}$ & $8 \times 10^{-2}$ \\
\hline 9 & 0 & 0 & $5 \times 10^{-4}$ & $2.5 \times 10^{-8}$ & $5 \times 10^{-2}$ & $2.5 \times 10^{-6}$ \\
\hline 10 & 0 & 0 & $5 \times 10^{-4}$ & $2.5 \times 10^{-8}$ & $5 \times 10^{-2}$ & $2.5 \times 10^{-6}$ \\
\hline 11 & 0 & 0 & $9 \times 10^{-4}$ & $4.5 \times 10^{-8}$ & $9 \times 10^{-2}$ & $4.5 \times 10^{-6}$ \\
\hline 12 & 0 & 0 & $10^{-3}$ & $10^{-3}$ & $10^{-1}$ & $10^{-1}$ \\
\hline 13 & 0 & 0 & $6 \times 10^{-4}$ & $3 \times 10^{-8}$ & $6 \times 10^{-2}$ & $3 \times 10^{-6}$ \\
\hline 14 & 0 & 0 & $7 \times 10^{-4}$ & $7 \times 10^{-4}$ & $7 \times 10^{-2}$ & $7 \times 10^{-2}$ \\
\hline
\end{tabular}

$(*)$ : in $\mathrm{m}^{3} / \mathrm{kg},(\dagger)$ : in $\mathrm{m}$ 


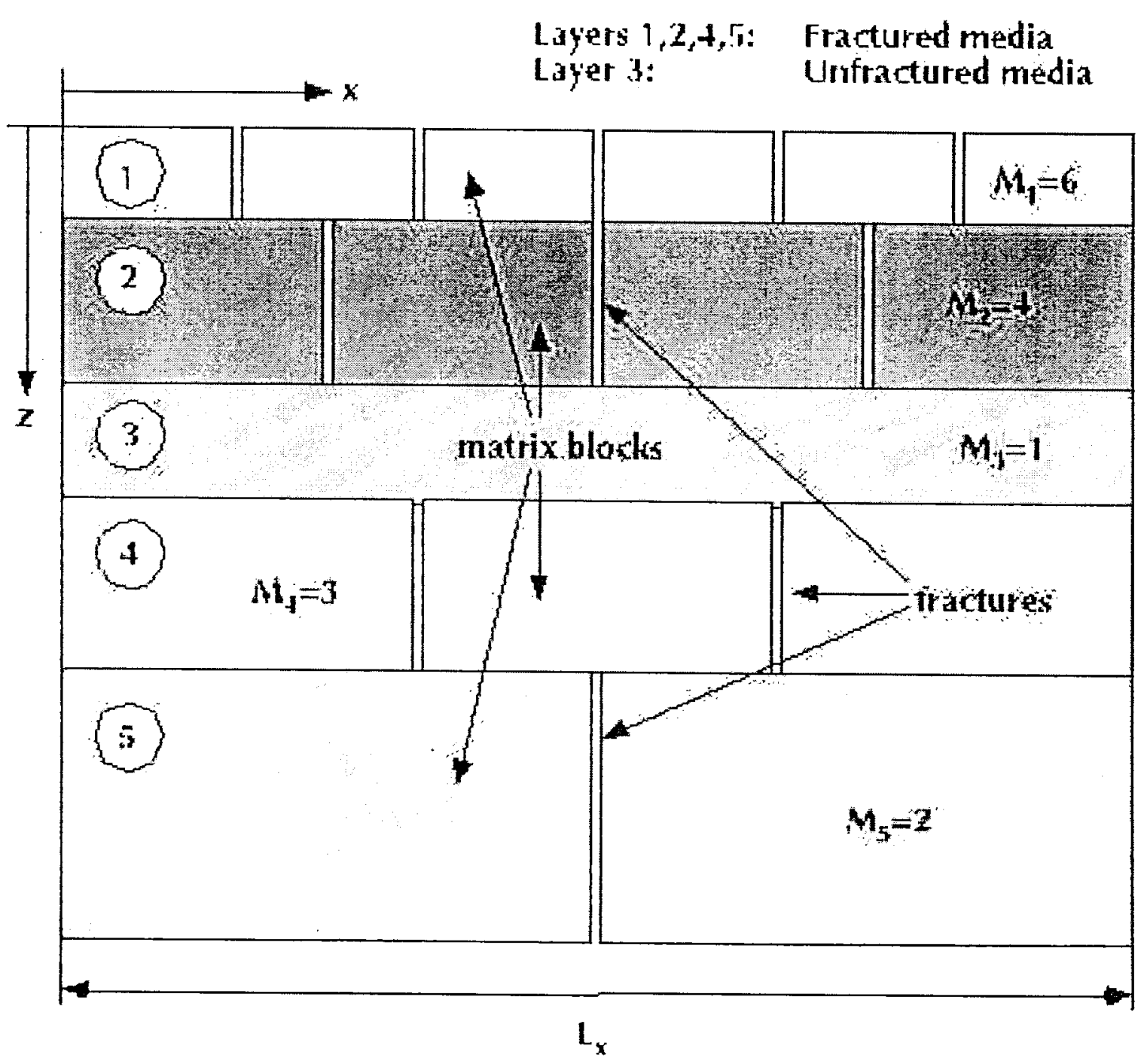

Figure 1. A variably-fractured layered geologic system. 


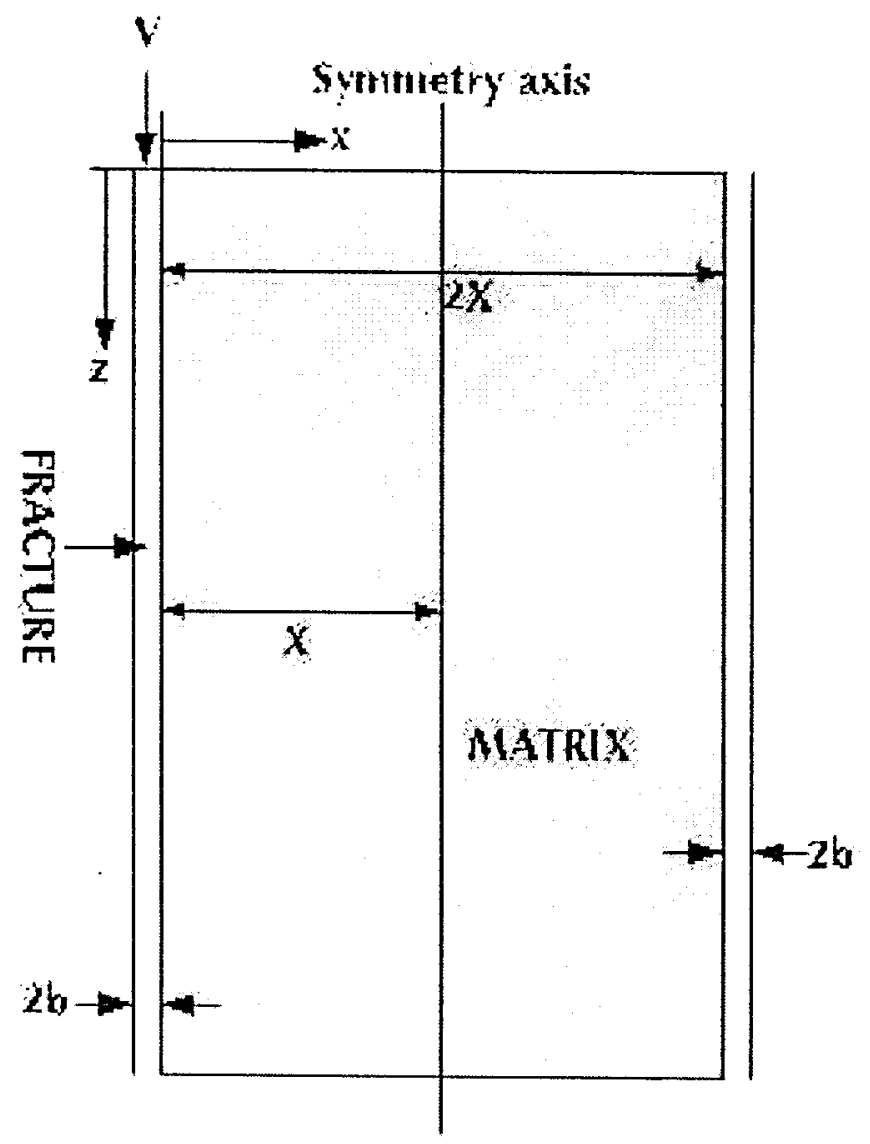

(a) Cas: 1

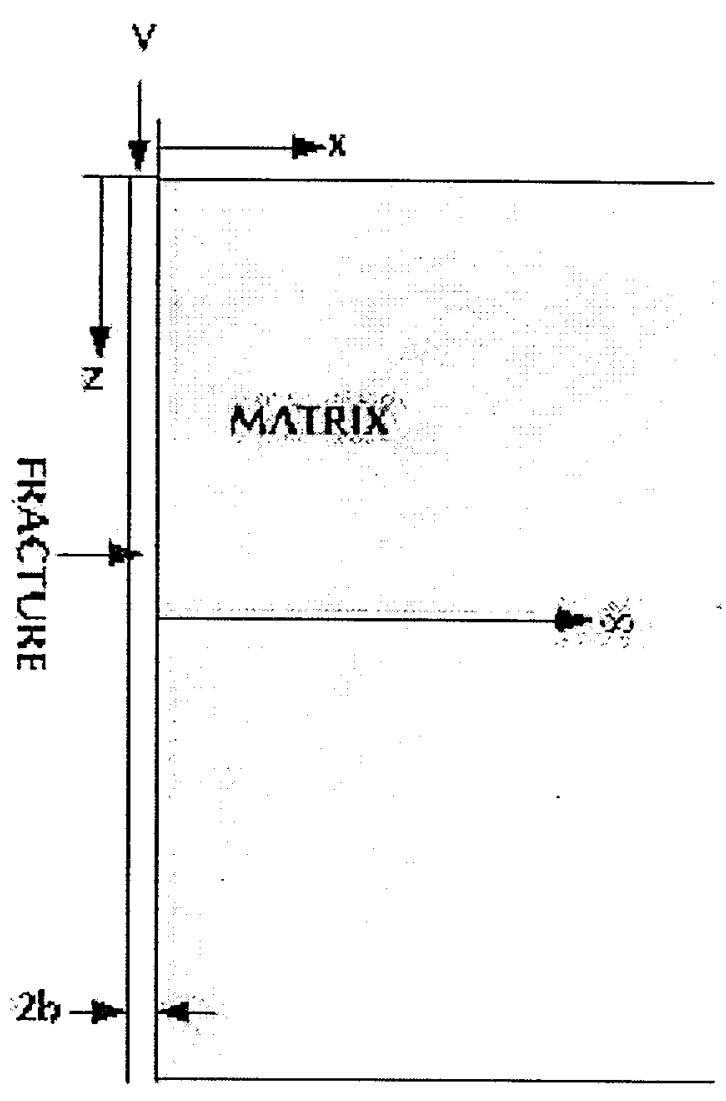

(k) C) C 2

Figure 2. Fracture-matrix configurations and important parameters in Cases 1 and 2. 


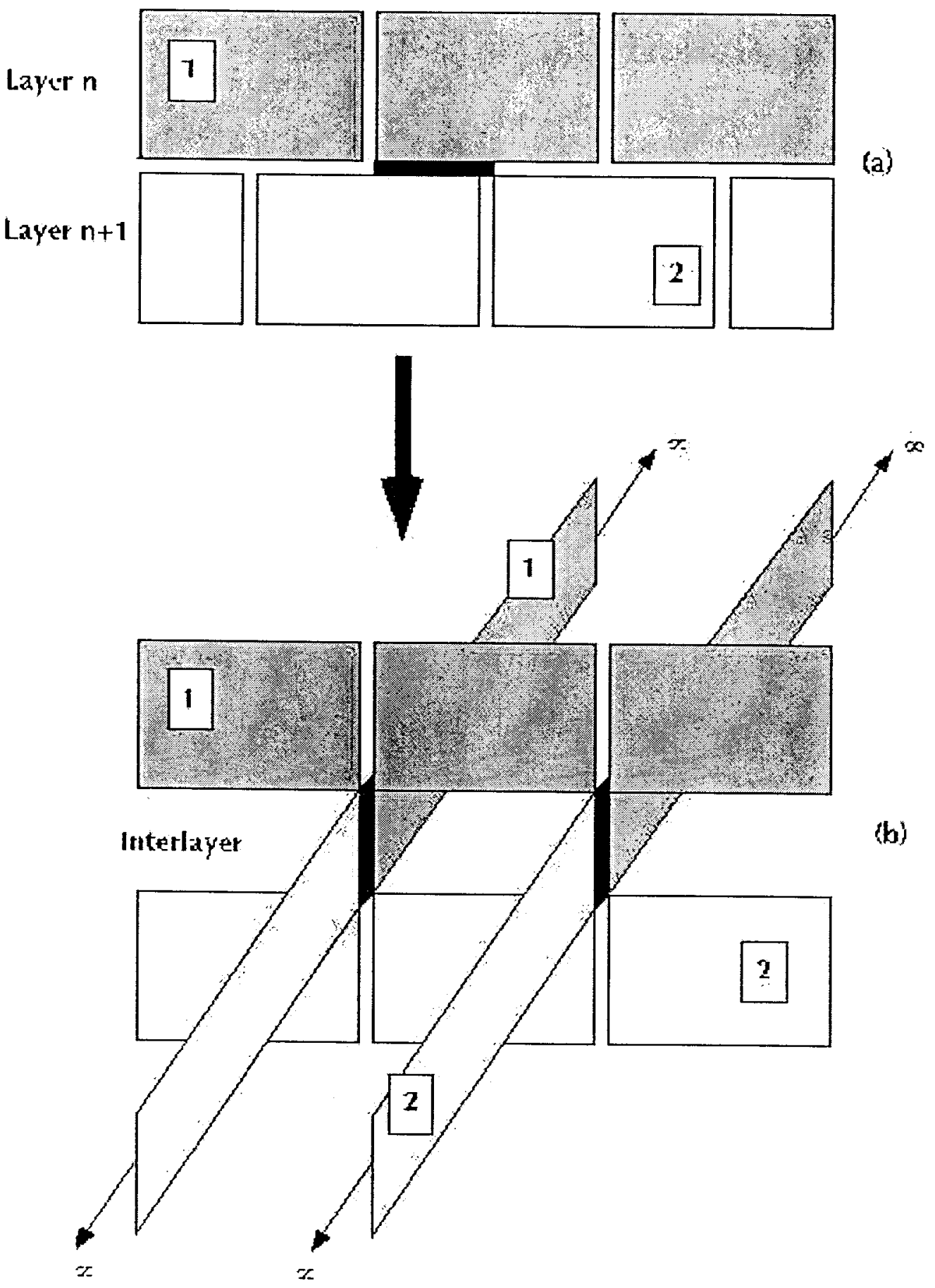

Figure 3. A graphic representation of the concept of interlayer describing the effects of fracture misalignment. The properties of layers $n$ and $n+1$ are denoted by 1 and 2 , respectively. 


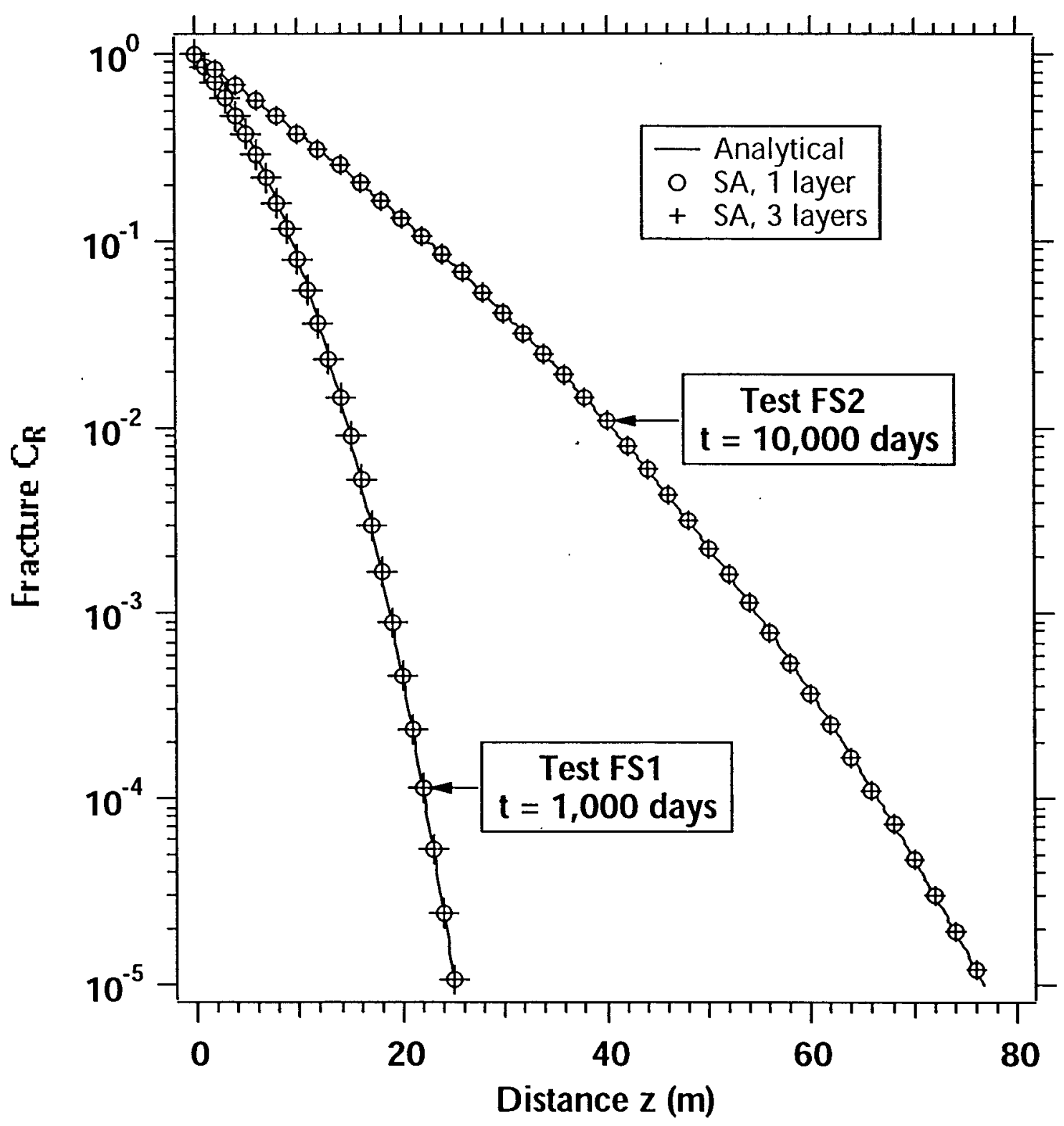

Figure 4. Comparison of the semianalytical (SA) solutions from FRACL to the analytical solution of radioactive solute transport in fractured media in Tests FS1 and FS2. 


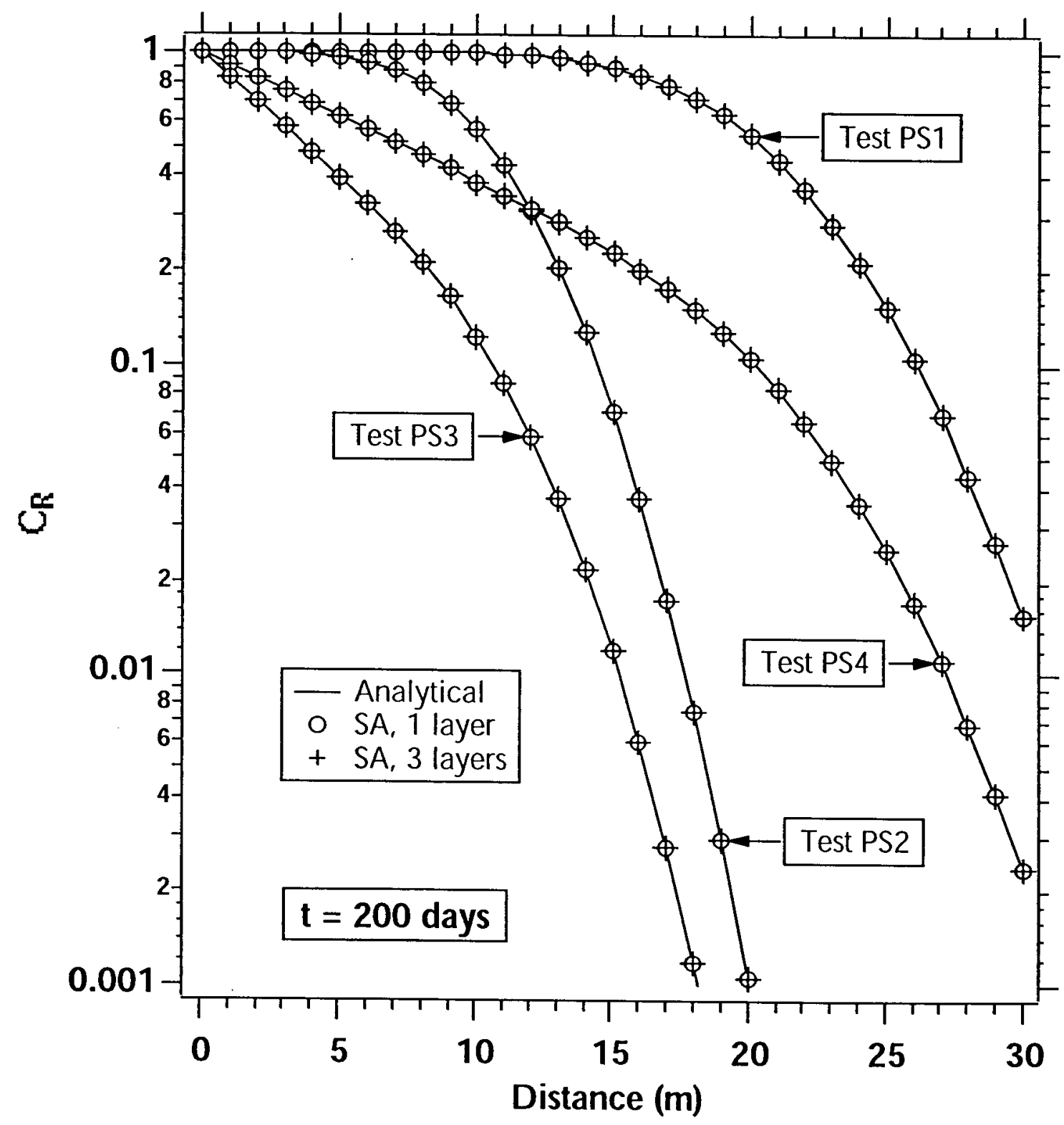

Figure 5. Comparison of the SA solutions to the analytical solutions of solute transport in porous media in Tests PS1 to PS4. 


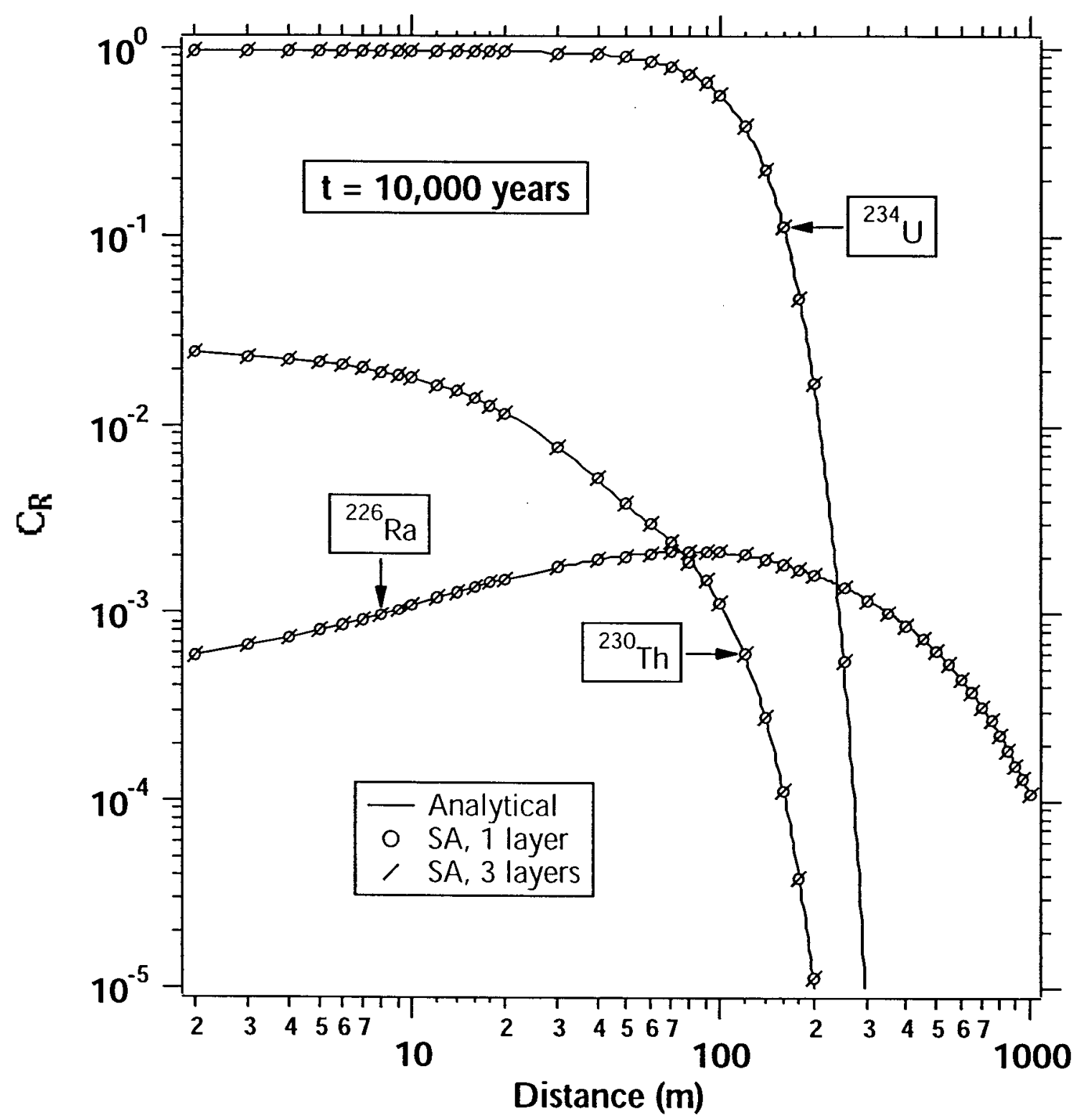

Figure 6. Comparison of the SA solutions from FRACL to the analytical solutions of solute transport of the radioactive chain ${ }^{234} \mathrm{U} \rightarrow{ }^{230} \mathrm{Th} \rightarrow{ }^{226} \mathrm{Ra}$ in porous media in Test PS5. 


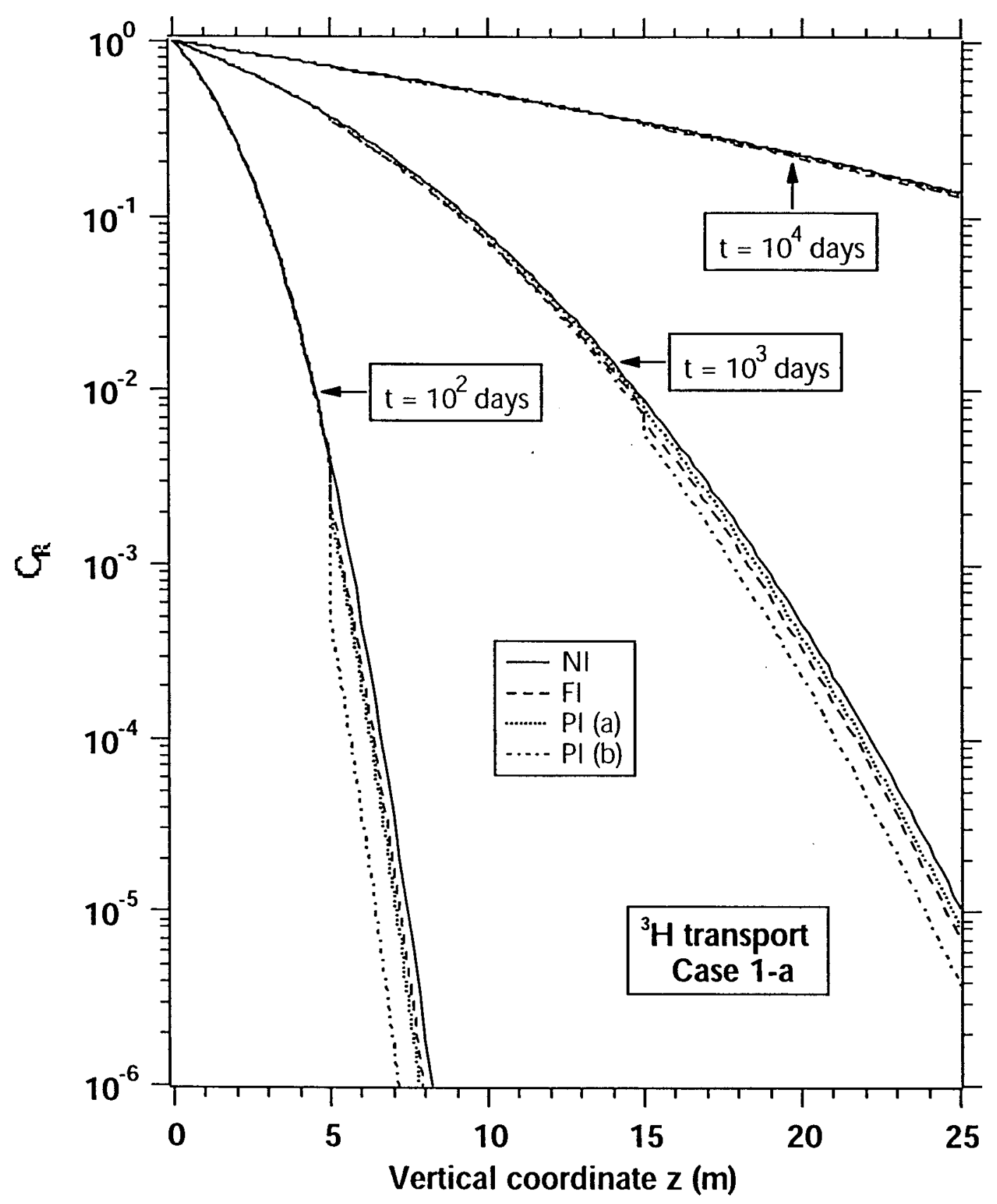

Figure 7. Effect of fracture offset (presence of interlayers) on the transport of ${ }^{3} \mathrm{H}$ through the layered fractured system of Case 1-a (NI: no interlayer, FI: fracture interlayer, PI(a): porous interlayer with $b=0.025 \mathrm{~m}, \mathrm{PI}(\mathrm{b})$ : porous interlayer with $b=0.1 \mathrm{~m})$. 


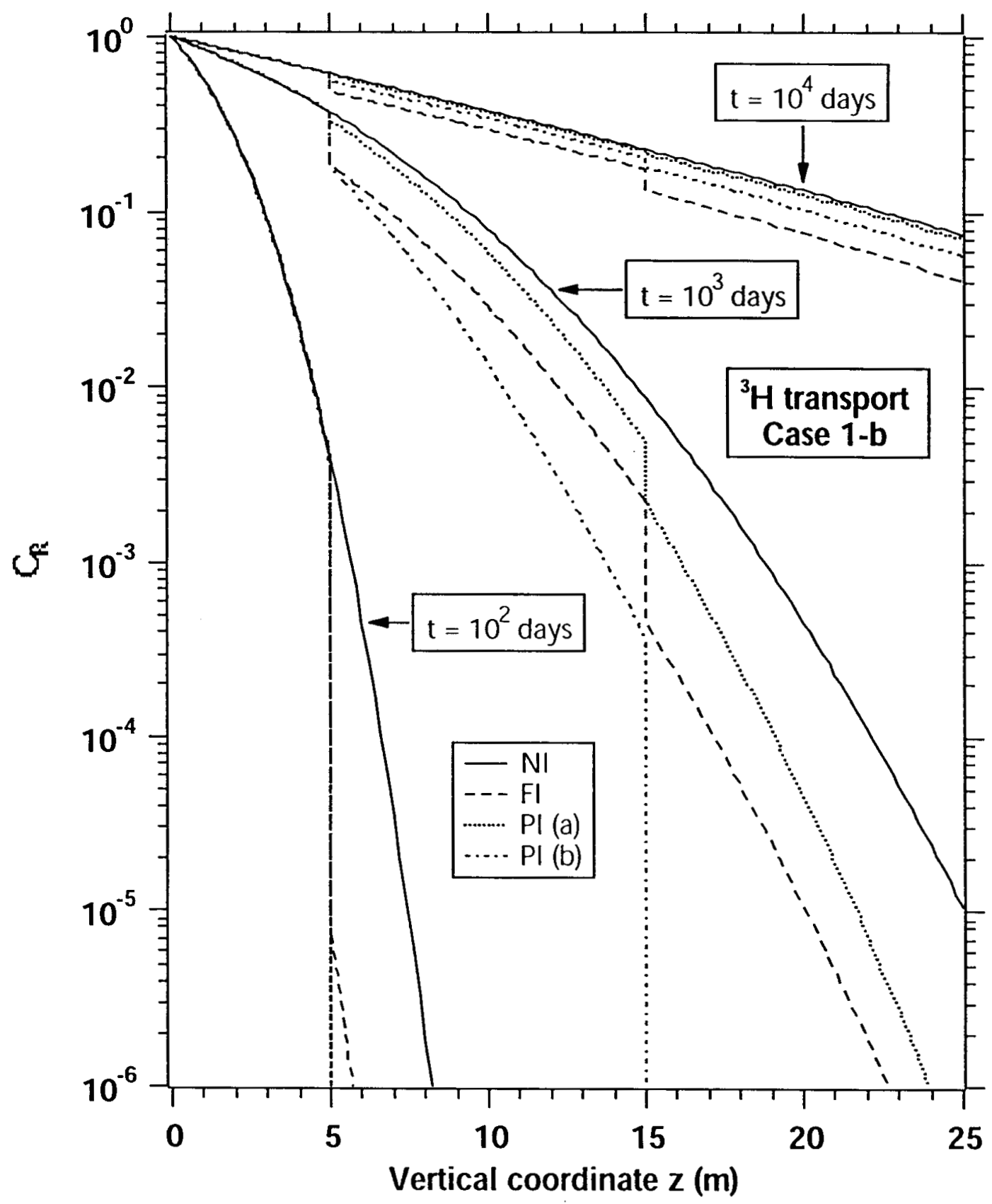

Figure 8. Combined effect of increased $X$ and fracture offset (presence of interlayers) on the transport of ${ }^{3} \mathrm{H}$ through the layered fractured system of Case 1-b (nomenclature as in Figure 7). 


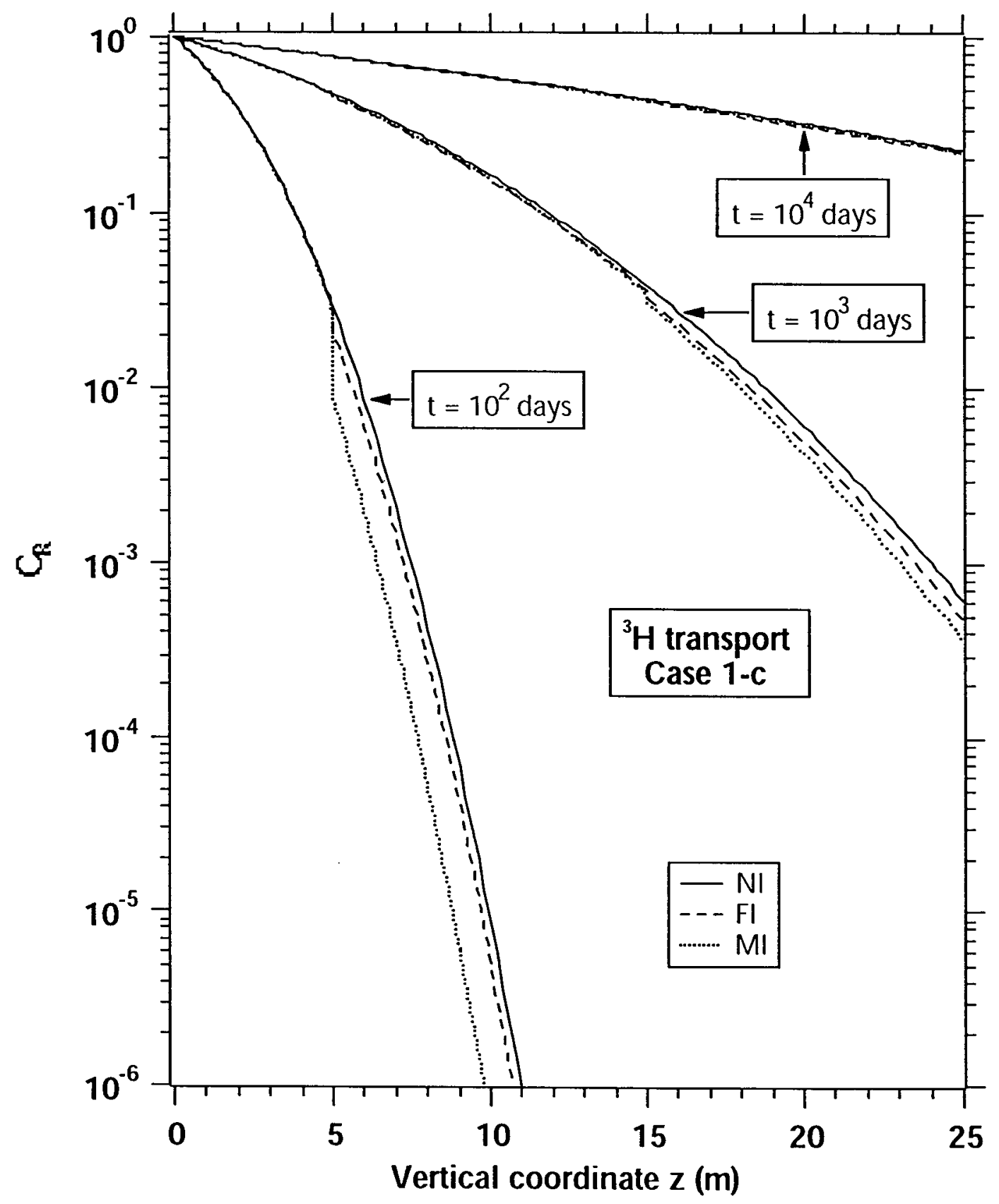

Figure 9. Combined effect of water saturation $S$ and fracture offset on the transport of ${ }^{3} \mathrm{H}$ through the layered fractured system of Case 1-c (nomenclature as in Figure 7). 


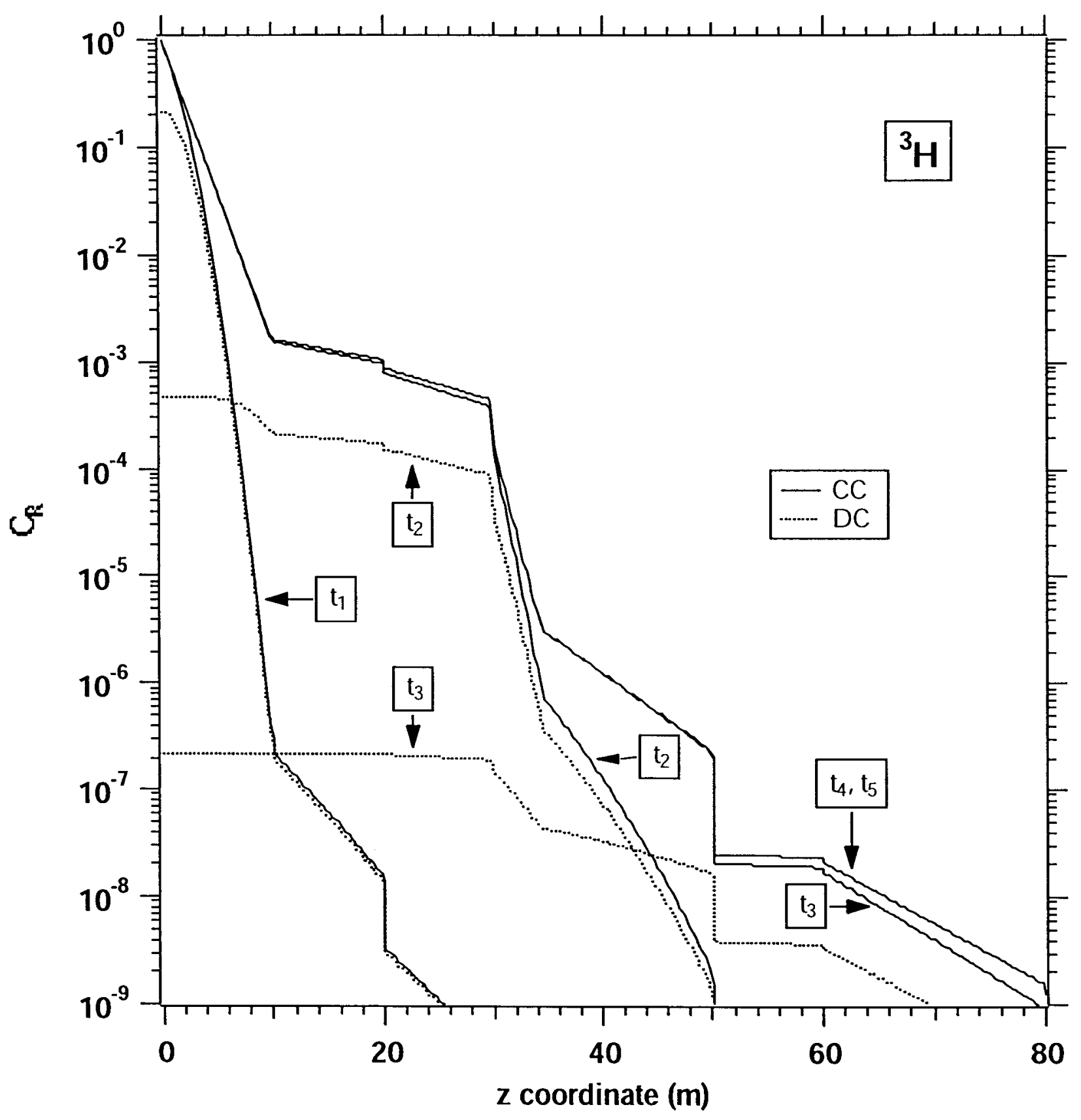

Figure 10. Fracture $C_{R}$ profiles of ${ }^{3} \mathrm{H}$ in the complex geological system of Problem 2 (CC: constant concentration boundary, DC: decaying concentration boundary). 


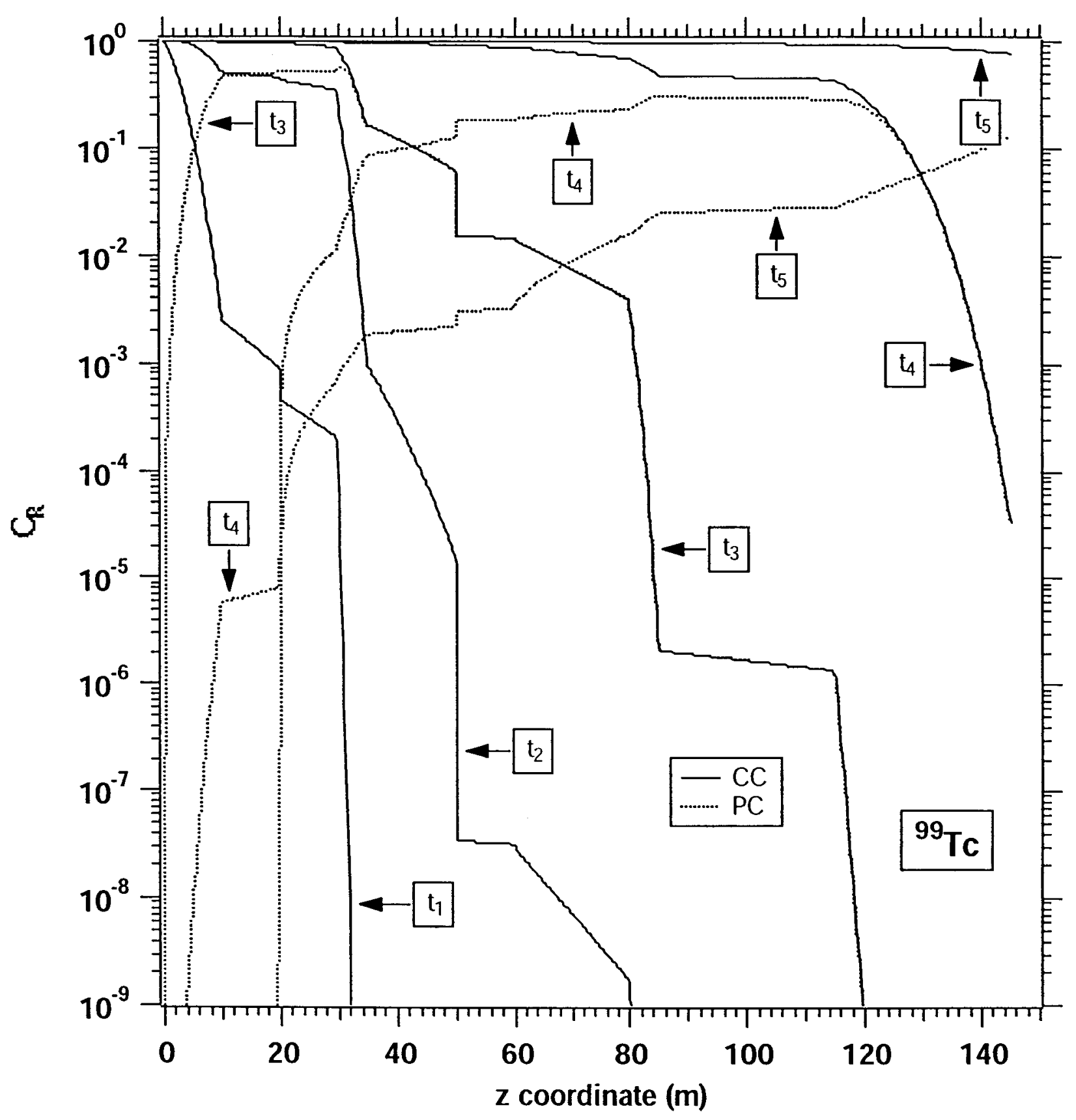

Figure 11. Fracture $C_{R}$ profiles of ${ }^{99} \mathrm{Tc}$ in the geological system of Problem 2 (PC: pulse concentration boundary). 


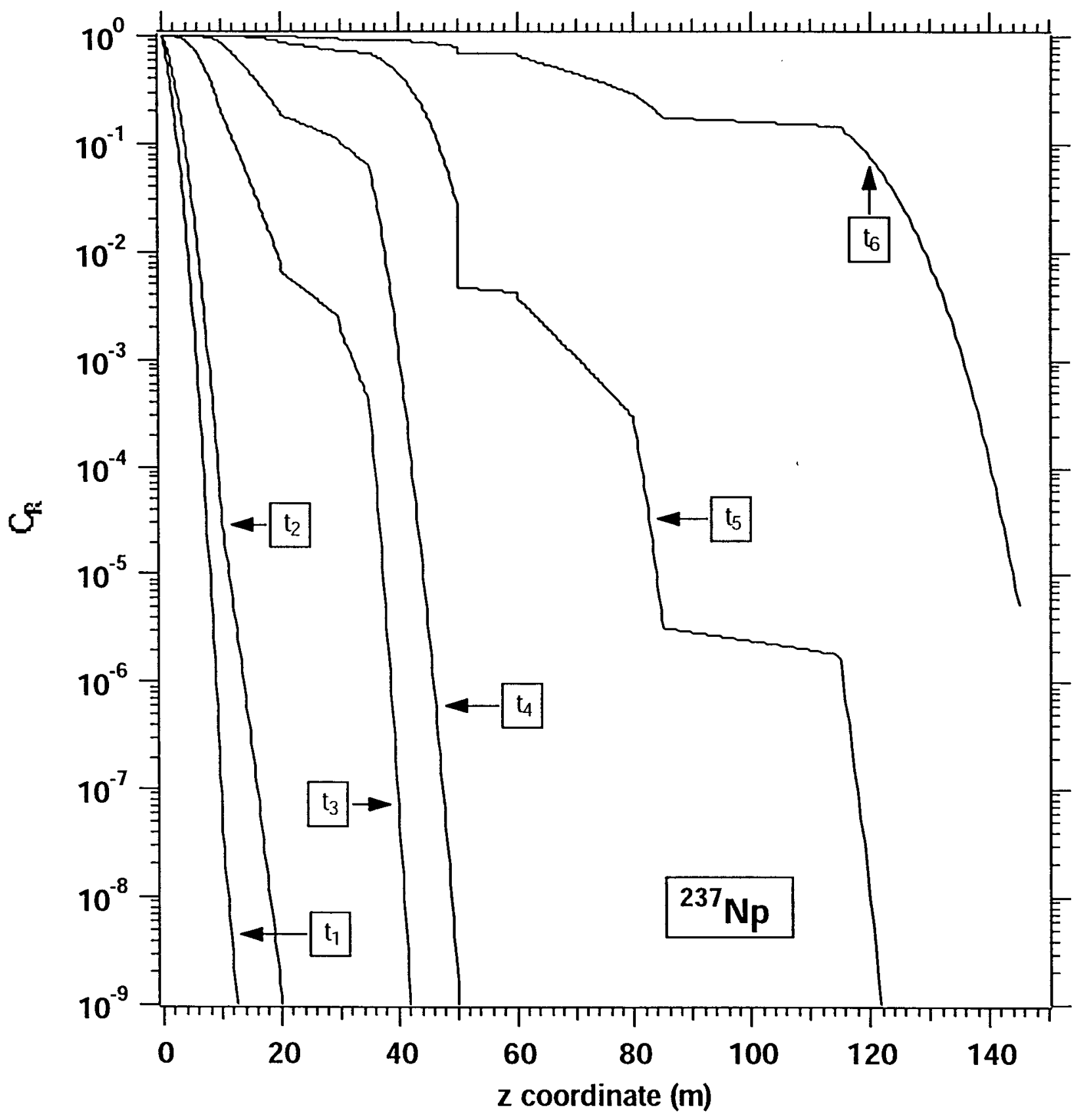

Figure 12. Fracture $C_{R}$ profiles of ${ }^{237} \mathrm{~Np}$ in the geological system of Problem 2 . 

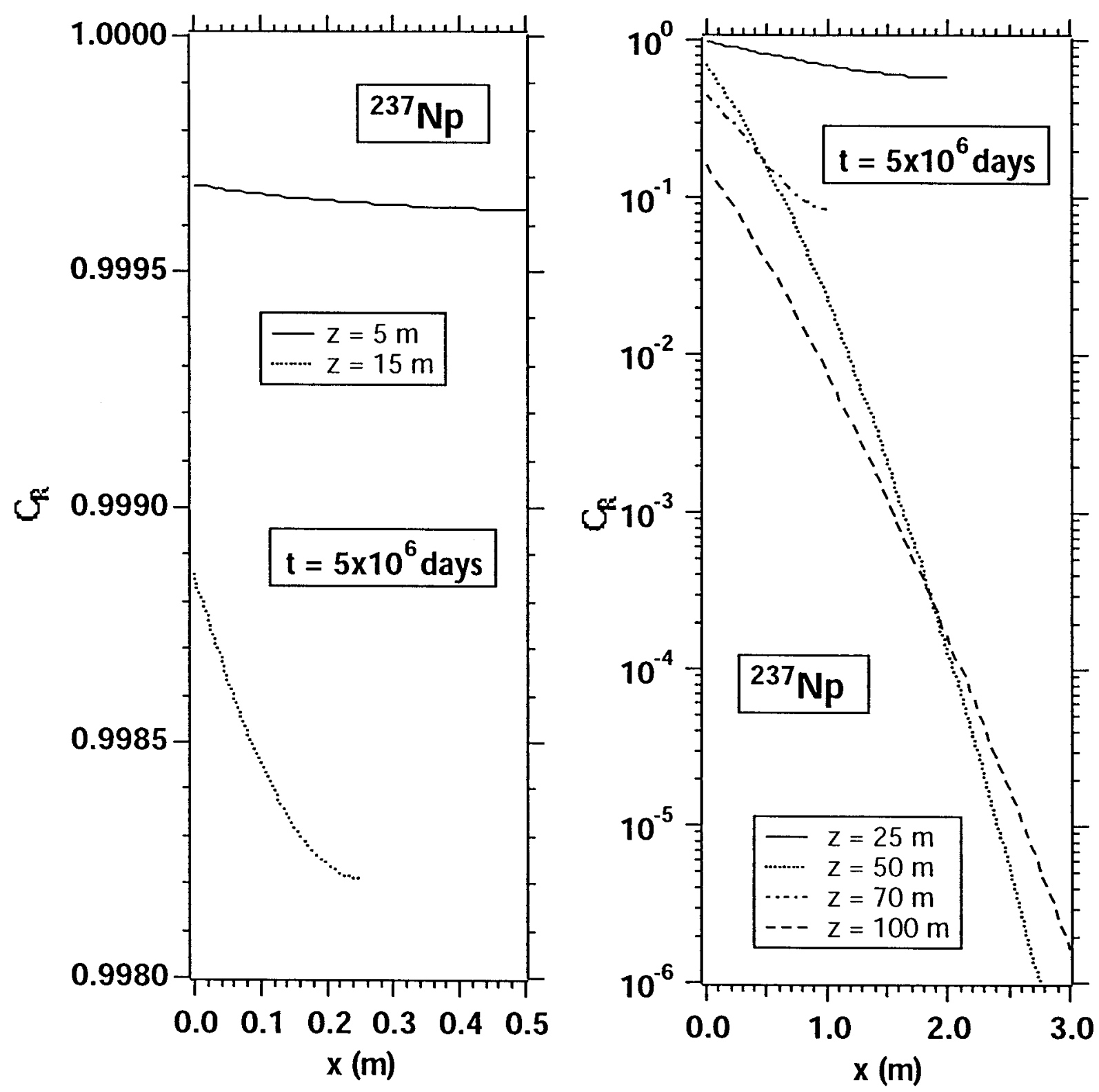

Figure 13. Matrix $C_{R}$ profiles of ${ }^{237} \mathrm{~Np}$ at different elevations in the geological system of Problem 2. 


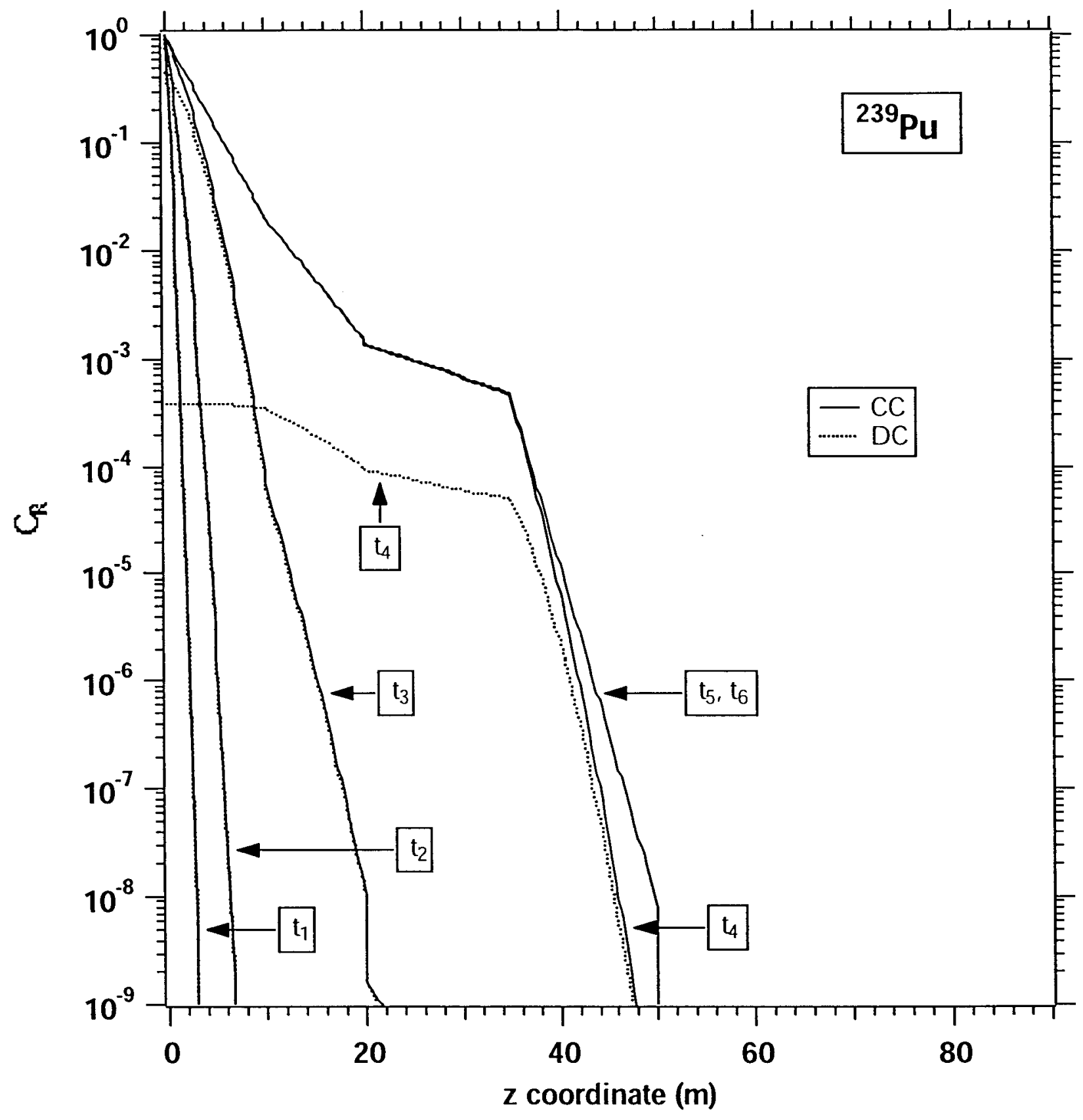

Figure 14. Fracture $C_{R}$ profiles of ${ }^{239} \mathrm{Pu}$ in the geological system of Problem 3. 


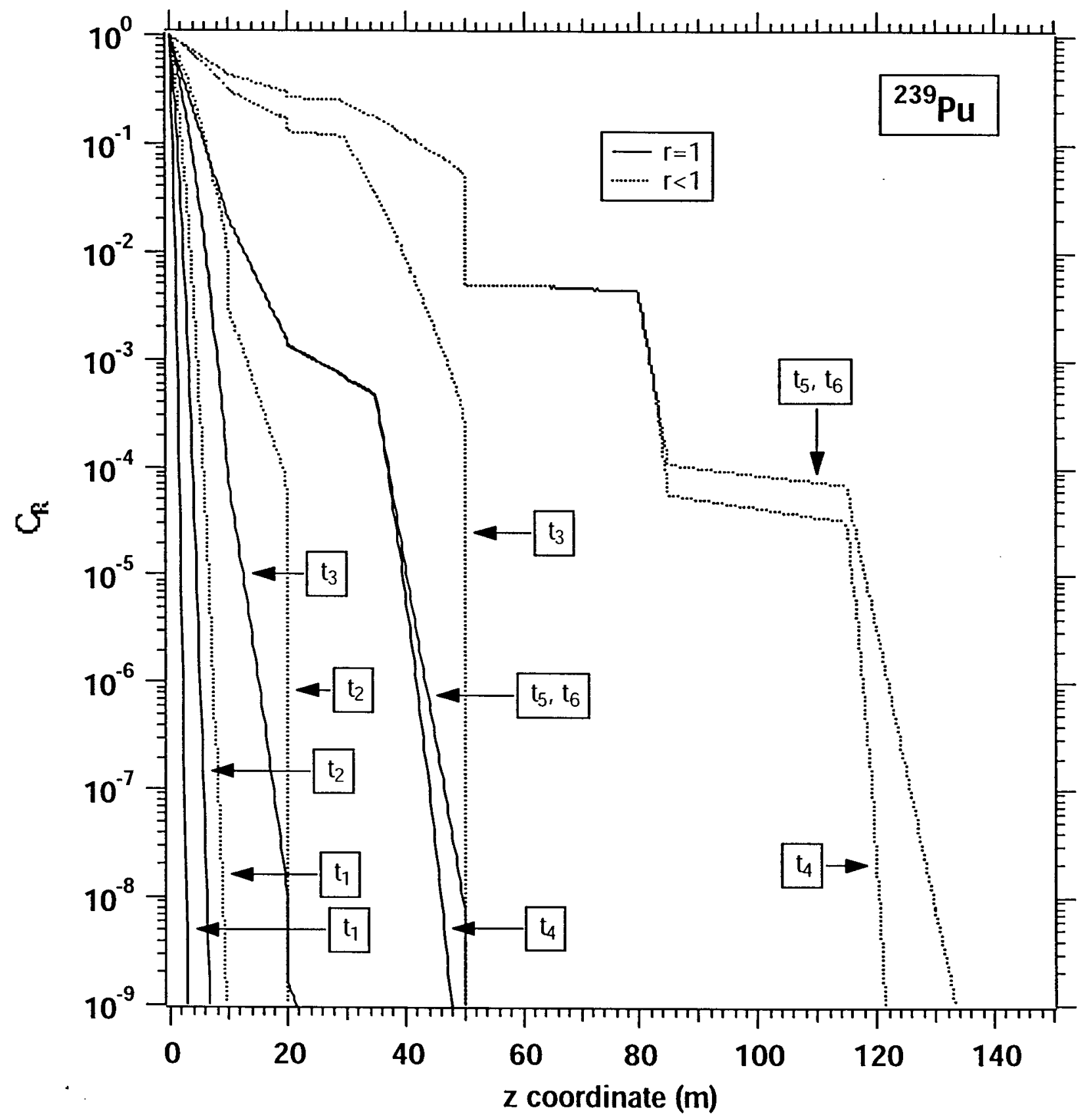

Figure 15. Effect of $r<1$ on the fracture $C_{R}$ profiles of ${ }^{239} \mathrm{Pu}$ in the geological system of Problem 3. 


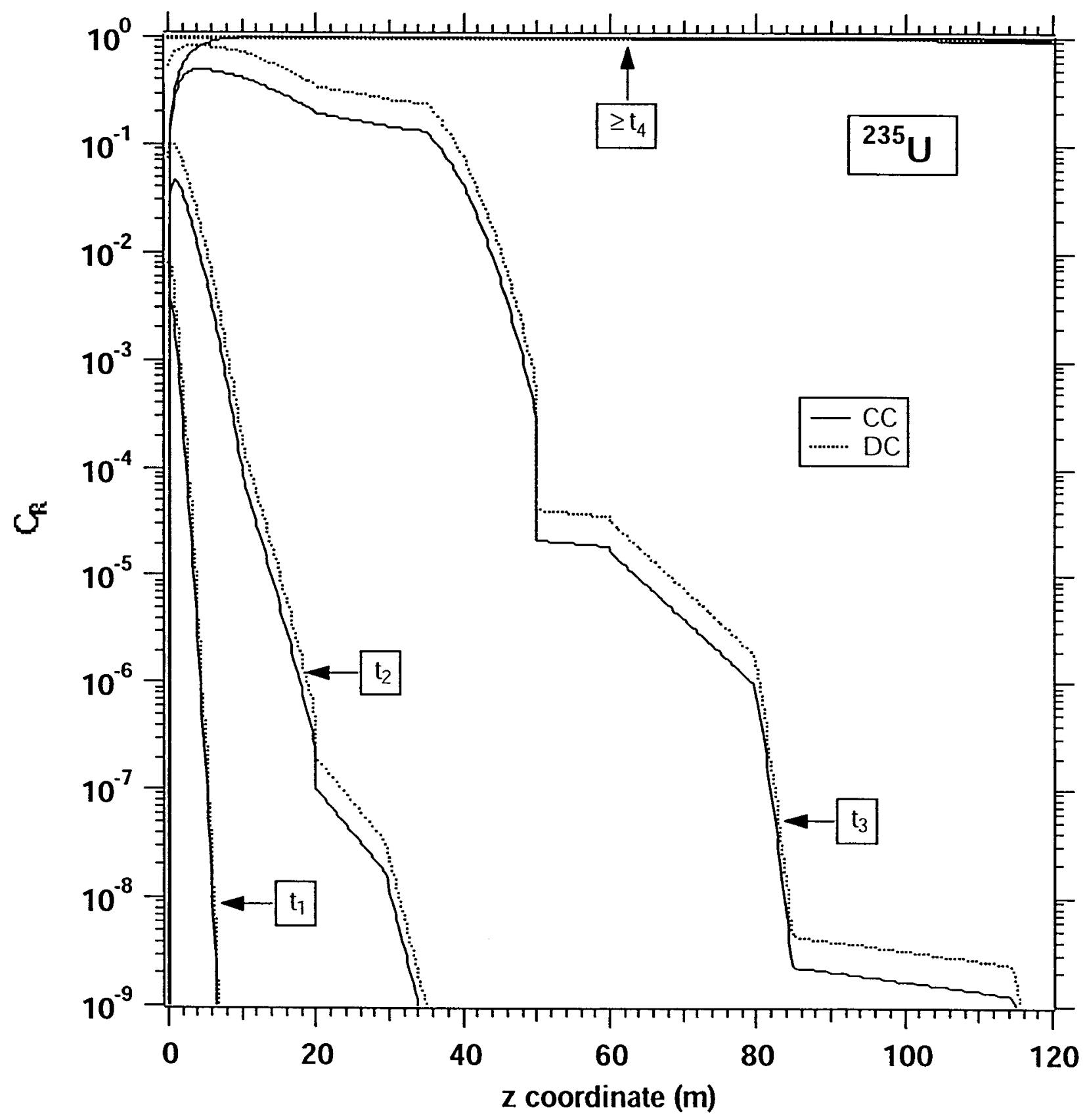

Figure 16. Fracture $C_{R}$ profiles of ${ }^{235} \mathrm{U}$ in the geological system of Problem 3 for $t \leq 10^{8}$ days. 


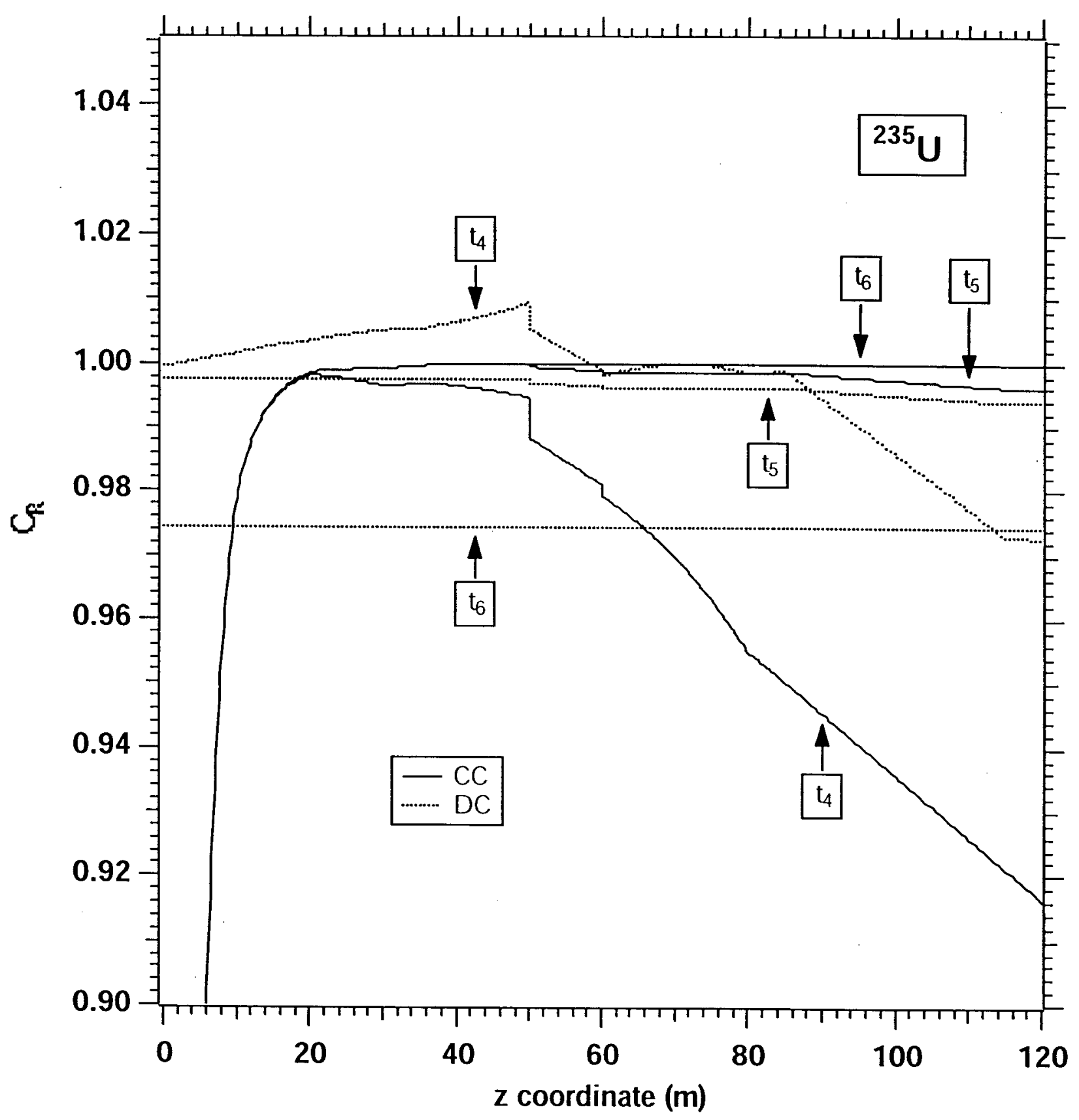

Figure 17. Fracture $C_{R}$ profiles of ${ }^{235} \mathrm{U}$ in the geological system of Problem 3 for $t \geq 10^{8}$ days. 


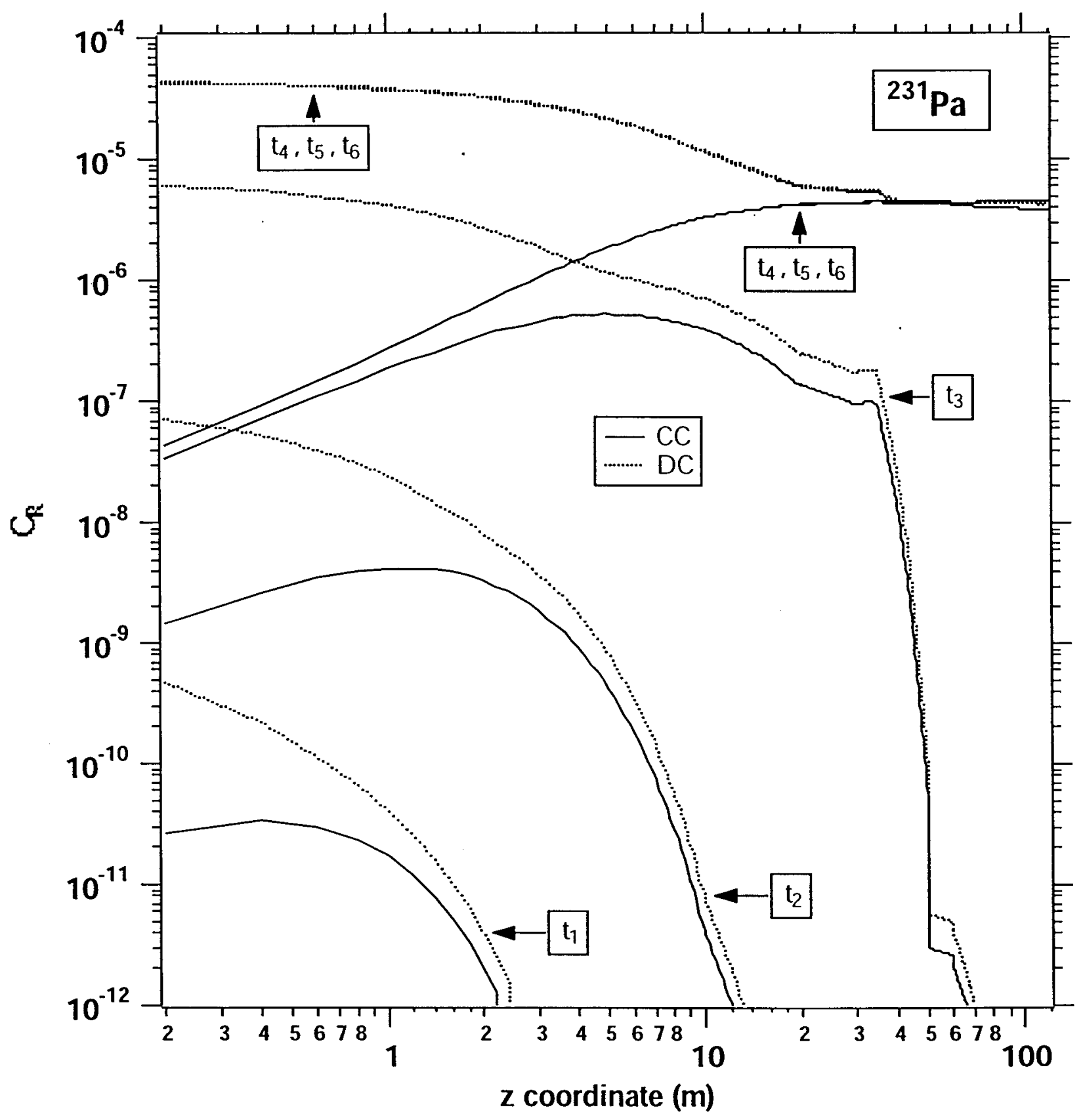

Figure 18. Fracture $C_{R}$ profiles of ${ }^{231} \mathrm{~Pa}$ in the geological system of Problem 3. 